\title{
Predictions for $B_{S} \rightarrow \bar{K}^{*} \ell \ell$ in non-universal $Z^{\prime}$ models
}

\author{
Ashutosh Kumar Alok ${ }^{1, a}$, Amol Dighe ${ }^{2, b}$, Shireen Gangal ${ }^{2, c}$, Dinesh Kumar ${ }^{3,4, d}$ \\ ${ }^{1}$ Indian Institute of Technology Jodhpur, Jodhpur 342037, India \\ 2 Tata Institute of Fundamental Research, Mumbai 400005, India \\ ${ }^{3}$ National Centre for Nuclear Research, Pasteura 7, 02-093 Warsaw, Poland \\ ${ }^{4}$ Department of Physics, University of Rajasthan, Jaipur 302004, India
}

Received: 28 March 2020 / Accepted: 11 July 2020 / Published online: 28 July 2020

(C) The Author(s) 2020

\begin{abstract}
The lepton flavor universality violating (LFUV) measurements $R_{K}$ and $R_{K^{*}}$ in $B$ meson decays can be accounted for in non-universal $Z^{\prime}$ models. We constrain the couplings of these $Z^{\prime}$ models by performing a global fit to correlated $b \rightarrow s \ell \ell$ and $b \rightarrow d \ell \ell$ processes, and calculate their possible implications for $B_{s} \rightarrow \bar{K}^{*} \ell \ell$ observables. For real new physics (NP) couplings, the $1 \sigma$ favored parameters allow the corresponding LFUV ratio $R_{K^{*}}^{(s)}$ in $B_{s} \rightarrow \bar{K}^{*} \ell \ell$ to range between 0.8 and 1.2 at low $q^{2}$. Complex NP couplings improve the best fit only marginally, however they allow a significant enhancement of the branching ratio, while increasing the range of $R_{K^{*}}^{(s)}$ at low $q^{2}$ to $0.8-1.8$. We find that NP could cause zero-crossing in the forward-backward asymmetry $A_{F B}$ to shift towards lower $q^{2}$ values, and enhancement in the magnitude of integrated $A_{F B}$. The $C P$ asymmetry $A_{C} P$ may be suppressed and even change sign. The simultaneous measurements of integrated $R_{K^{*}}^{(s)}$ and $A_{C P}$ values to 0.1 and $1 \%$ respectively, would help in constraining the effective NP Wilson coefficient $C_{9}$ in $b \rightarrow d \mu \mu$ interactions.
\end{abstract}

\section{Introduction}

In recent times, the most tenacious hints of physics beyond the standard model (SM) have been seen in the decays of $B$ mesons. In particular, there are several measurements in the decays involving the quark-level transition $b \rightarrow s \ell^{+} \ell^{-}$ $(l=e, \mu)$ that deviate from the predictions of SM. These include the LFUV observables $R_{K}$ and $R_{K^{*}}$ [1,2] whose measurements disagree with the SM predictions at the level of $\sim 2.5 \sigma[3,4]$. This disagreement can be attributed to NP in $b \rightarrow s e^{+} e^{-}$and/or $b \rightarrow s \mu^{+} \mu^{-}$[5-7]. There are also

\footnotetext{
a e-mail: akalok@iitj.ac.in

be-mail: amol@theory.tifr.res.in

c e-mail: shireen.gangal@theory.tifr.res.in (corresponding author)

d e-mail: dinesh.kumar@ncbj.gov.pl
}

deviations from the SM expectations at the level of $\sim 4 \sigma$ in other measurements involving only $b \rightarrow s \mu^{+} \mu^{-}$transition, such as the branching ratio of $B_{s} \rightarrow \phi \mu^{+} \mu^{-}[8]$ and angular observable $P_{5}^{\prime}$ in $B \rightarrow K^{*} \mu^{+} \mu^{-}$decay [9-11]. Hence it is natural to try accounting for the discrepancies in all the above measurements by assuming new physics only in the muon sector.

These anomalies may be addressed in a model-agnostic way using the framework of effective field theory, where the effects of NP are incorporated by adding new operators to the SM effective Hamiltonian. Various groups have performed global fits to all available data in the $b \rightarrow s \ell^{+} \ell^{-}$sector in order to identify the Lorentz structure of possible new physics operators [12-21]. Some of these new physics operators can be generated in $Z^{\prime}$ [22-26] or leptoquark models [27-32]. It has been shown that several models with $Z^{\prime}$, either light or heavy, can help account for the anomalies in $b \rightarrow s \mu^{+} \mu^{-}$ sector [33-39].

Since the $Z^{\prime}$ boson would in general couple to all generations, the imprints of such a $Z^{\prime}$ would be seen in other flavor sectors as well. Therefore, it is worth extending this model to include other related decays. This will provide further insights into the NP flavor structure. In this work, we consider possible observable effects of $Z^{\prime}$ models on decays induced by the quark-level transition $b \rightarrow d \mu^{+} \mu^{-}$.

The $b \rightarrow d \mu^{+} \mu^{-}$transition gives rise to inclusive semileptonic decays $\bar{B} \rightarrow X_{d} \mu^{+} \mu^{-}$as well as exclusive semileptonic decays such as $\bar{B} \rightarrow\left(\pi^{0}, \rho\right) \mu^{+} \mu^{-}, B^{+} \rightarrow$ $\pi^{+} \mu^{+} \mu^{-}$, and $B_{s} \rightarrow \bar{K}^{*} \mu^{+} \mu^{-}$. Till recently, the only observed decay mode among these was $B^{+} \rightarrow \pi^{+} \mu^{+} \mu^{-}$ $[40,41]$, however now LHCb has reported an evidence for the decay $B_{s} \rightarrow \bar{K}^{*} \mu^{+} \mu^{-}$with a measured branching ratio of $(2.9 \pm 1.1) \times 10^{-8}$ [42]. For other decays, we only have an upper bound on their branching ratios $[43,44]$.

A large number of $b \rightarrow d \mu^{+} \mu^{-}$decays, at the level of thousands or tens of thousands, would be observed after the 
LHC upgrade. For example, about 17,000 $B^{+} \rightarrow \pi^{+} \mu^{+} \mu^{-}$ events are expected to be observed after collection of the full $300 \mathrm{fb}^{-1}$ dataset. For $B_{S} \rightarrow \bar{K}^{*} \mu^{+} \mu^{-}$decays, the full angular analysis is expected to be possible after the LHCb Upgrade-II dataset, where around 4300 events could be observed [45]. This would enable the measurements of angular observables in $B_{s} \rightarrow \bar{K}^{*} \mu^{+} \mu^{-}$decays with a precision even better than the existing measurements of angular distributions in $B_{d} \rightarrow K^{*} \mu^{+} \mu^{-}$decay.

Currently, as there are not many measurements in the $b \rightarrow d$ sector, a model-independent analysis would not be very useful in constraining new physics. However, in the context of specific models (like $Z^{\prime}$ ), some of the couplings can be constrained from the $b \rightarrow s$ sector and neutrino trident production. Therefore we choose this approach to constrain the effective couplings in the $b \rightarrow d \mu^{+} \mu^{-}$sector, and identify potential observables in the $B_{s} \rightarrow \bar{K}^{*} \mu^{+} \mu^{-}$decay where large new physics effects are possible.

The paper is organized as follows. In Sect. 2, we introduce the $Z^{\prime}$ model considered in this work and indicate how it can be constrained by available measurements. We then describe our fit methodology in Sect. 3. The fit results along with predictions of various $B_{S} \rightarrow \bar{K}^{*} \mu^{+} \mu^{-}$observables are presented in Sect. 4. We summarize in Sect. 5.

\section{The $Z^{\prime}$ model and sources of constraints}

In the non-universal $Z^{\prime}$ model that we consider, the $Z^{\prime}$ boson is associated with a new $U(1)^{\prime}$ symmetry. It couples to both left-handed and right-handed muons but not to leptons of other generations. It couples to both left-handed and righthanded quarks, however we assume its couplings to righthanded quarks to be flavor-diagonal, thereby avoiding contribution of new chirality flipped operators to flavor changing neutral current (FCNC) decays $[46,47]$. The change in the Lagrangian density due to the addition of such a heavy $Z^{\prime}$ boson is

$\Delta \mathcal{L}_{Z^{\prime}}=J^{\alpha} Z_{\alpha}^{\prime}$,

where

$$
\begin{aligned}
J^{\alpha} \supset & g_{L}^{\mu \mu} \bar{L} \gamma^{\alpha} P_{L} L+g_{R}^{\mu \mu} \bar{L} \gamma^{\alpha} P_{R} L+g_{L}^{b d} \bar{Q}_{1} \gamma^{\alpha} P_{L} Q_{3} \\
& +g_{L}^{b s} \bar{Q}_{2} \gamma^{\alpha} P_{L} Q_{3}+\text { h.c. }
\end{aligned}
$$

The right-hand side in Eq. (2) includes only the terms contributing to FCNC processes. Here $P_{L(R)}=\left(1 \mp \gamma_{5}\right) / 2, Q_{i}$ is the $i^{t h}$ generation of quark doublet, and $L=\left(v_{\mu}, \mu\right)^{T}$ is the second generation doublet. Further, $g_{L(R)}^{\mu \mu}$ are the left-handed (right-handed) couplings of the $Z^{\prime}$ boson to muons, and $g_{L}^{b q}$ to quarks. One can integrate out the heavy $Z^{\prime}$ and get the relevant terms in the effective four-fermion Hamiltonian as,

$$
\begin{aligned}
\mathcal{H}_{\mathrm{eff}}^{Z^{\prime}} & =\frac{1}{2 M_{Z^{\prime}}^{2}} J_{\alpha} J^{\alpha} \\
\supset & \frac{g_{L}^{b s}}{M_{Z^{\prime}}^{2}}\left(\bar{s} \gamma^{\alpha} P_{L} b\right)\left[\bar{\mu} \gamma_{\alpha}\left(g_{L}^{\mu \mu} P_{L}+g_{R}^{\mu \mu} P_{R}\right) \mu\right] \\
& +\frac{\left(g_{L}^{b s}\right)^{2}}{2 M_{Z^{\prime}}^{2}}\left(\bar{s} \gamma^{\alpha} P_{L} b\right)\left(\bar{s} \gamma_{\alpha} P_{L} b\right) \\
& +\frac{g_{L}^{b d}}{M_{Z^{\prime}}^{2}}\left(\bar{d} \gamma^{\alpha} P_{L} b\right)\left[\bar{\mu} \gamma_{\alpha}\left(g_{L}^{\mu \mu} P_{L}+g_{R}^{\mu \mu} P_{R}\right) \mu\right] \\
& +\frac{\left(g_{L}^{b d}\right)^{2}}{2 M_{Z^{\prime}}^{2}}\left(\bar{d} \gamma^{\alpha} P_{L} b\right)\left(\bar{d} \gamma_{\alpha} P_{L} b\right) \\
& +\frac{g_{L}^{\mu \mu}}{M_{Z^{\prime}}^{2}}\left(\bar{v}_{\mu} \gamma_{\alpha} P_{L} v_{\mu}\right)\left[\bar{\mu} \gamma^{\alpha}\left(g_{L}^{\mu \mu} P_{L}+g_{R}^{\mu \mu} P_{R}\right) \mu\right],
\end{aligned}
$$

where we have taken the down-type quarks in the quarkdoublets $Q_{i}$ to be in the mass-flavor diagonal basis. In Eq. (3), the first (third) term corresponds to $b \rightarrow s(d) \mu^{+} \mu^{-}$transitions, the second (fourth) terms give rise to $B_{s}-\bar{B}_{s}\left(B_{d}-\bar{B}_{d}\right)$ mixing, whereas the fifth term contributes to the neutrino trident production $v_{\mu} N \rightarrow v_{\mu} N \mu^{+} \mu^{-}(N=$ nucleus). Consequently, the products $g_{L}^{b s} g_{L, R}^{\mu \mu}\left(g_{L}^{b d} g_{L, R}^{\mu \mu}\right)$ are constrained by the $b \rightarrow s(d) \mu^{+} \mu^{-}$data, and individual magnitudes $\left|g_{L}^{b s}\right|$ $\left(\left|g_{L}^{b d}\right|\right)$ from the $B_{s}-\bar{B}_{s}\left(B_{d}-\bar{B}_{d}\right)$ mixing. The neutrino trident production puts limits on the individual muon couplings $g_{L, R}^{\mu \mu}$. We now discuss constraints on the $Z^{\prime}$ couplings arising from each of the above measurements.

\section{$2.1 b \rightarrow s(d) \mu^{+} \mu^{-}$decays}

The effective Hamiltonian for $b \rightarrow q \mu^{+} \mu^{-}$transition in the $\mathrm{SM}$ is

$$
\begin{aligned}
\mathcal{H}_{\mathrm{eff}}^{\mathrm{SM}}= & -\frac{4 G_{F}}{\sqrt{2}} V_{t q}^{*} V_{t b}\left[\sum_{i=1}^{6} C_{i} \mathcal{O}_{i}\right. \\
& +C_{7}^{b q} \frac{e}{16 \pi^{2}}\left[\bar{q} \sigma_{\mu \nu}\left(m_{q} P_{L}+m_{b} P_{R}\right) b\right] F^{\mu \nu}+C_{8}^{b q} \mathcal{O}_{8} \\
& +C_{9}^{b q, \mathrm{SM}} \frac{\alpha_{\mathrm{em}}}{4 \pi}\left(\bar{q} \gamma^{\mu} P_{L} b\right)\left(\bar{\mu} \gamma_{\mu} \mu\right) \\
& \left.+C_{10}^{b q, \mathrm{SM}} \frac{\alpha_{\mathrm{em}}}{4 \pi}\left(\bar{q} \gamma^{\mu} P_{L} b\right)\left(\bar{\mu} \gamma_{\mu} \gamma_{5} \mu\right)\right]
\end{aligned}
$$

where $G_{F}$ is the Fermi constant and $V_{i j}$ are the CabibboKobayashi-Maskawa (CKM) matrix elements. The Wilson coefficients (WC) $C_{i}$ of the four-fermi operators $\mathcal{O}_{i}$ encode the short-distance contributions to the Hamiltonian in the $\mathrm{SM}$, where the scale-dependence is implicit, i.e. $C_{i} \equiv C_{i}(\mu)$ and $\mathcal{O}_{i} \equiv \mathcal{O}_{i}(\mu)$. The operators $\mathcal{O}_{i}(i=1, \ldots, 6,8)$ contribute to these processes through the modifications $C_{7,9}(\mu)$ $\rightarrow C_{7.9}^{\text {eff }}\left(\mu, q^{2}\right)$, where $q^{2}$ is the invariant mass-squared of the final state muon pair. We drop the superscript "eff" 
from here on for the sake of brevity. Addition of the new $Z^{\prime}$ boson to the SM particle spectrum modifies the WCs as $C_{9,10}^{b q} \rightarrow C_{9,10}^{b q, \mathrm{SM}}+C_{9,10}^{b q, \mathrm{NP}}$, where

$$
\begin{aligned}
C_{9}^{b q, \mathrm{NP}} & =-\frac{\pi}{\sqrt{2} G_{F} \alpha V_{t b} V_{t q}^{*}} \frac{g_{L}^{b q}\left(g_{L}^{\mu \mu}+g_{R}^{\mu \mu}\right)}{M_{Z^{\prime}}^{2}}, \\
C_{10}^{b q, \mathrm{NP}} & =\frac{\pi}{\sqrt{2} G_{F} \alpha V_{t b} V_{t q}^{*}} \frac{g_{L}^{b q}\left(g_{L}^{\mu \mu}-g_{R}^{\mu \mu}\right)}{M_{Z^{\prime}}^{2}} .
\end{aligned}
$$

In the $Z^{\prime}$ models, $C_{9}^{b s, \mathrm{NP}}$ and $C_{10}^{b s, \mathrm{NP}}$ are in general independent. Two of the one-parameter scenarios, $C_{10}^{b s, \mathrm{NP}}=0$ (popularly known as $C_{9}^{b s, \mathrm{NP}}<0$ ) and $C_{9}^{b s, \mathrm{NP}}=-C_{10}^{b s, \mathrm{NP}}$, can be realized by substituting $g_{L}^{\mu \mu}=g_{R}^{\mu \mu}$ and $g_{R}^{\mu \mu}=0$, respectively.

\section{$2.2 B_{s(d)}-\bar{B}_{s(d)}$ mixing}

The dominant contribution to $B_{q}-\bar{B}_{q}$ mixing within the SM comes from the virtual top quark in the box diagram. The $Z^{\prime}$ boson contributes to $B_{q}-\bar{B}_{q}$ mixing at the tree-level. The combined contribution to $M_{12}^{q}$, the dispersive part of the box diagrams responsible for the mixing, is

$M_{12}^{q}=\frac{1}{3} M_{B_{q}} f_{B_{q}}^{2} \widehat{B}_{B_{q}}\left[N C_{\mathrm{VLL}}^{\mathrm{SM}}+\frac{\left(g_{L}^{b q}\right)^{2}}{2 M_{Z^{\prime}}^{2}}\right]$,

where

$$
\begin{aligned}
N & =\frac{G_{F}^{2} M_{W}^{2}}{16 \pi^{2}}\left(V_{t b} V_{t q}^{*}\right)^{2}, \\
C_{\mathrm{VLL}}^{\mathrm{SM}} & =\eta_{B} x_{t}\left[1+\frac{9}{1-x_{t}}-\frac{6}{\left(1-x_{t}\right)^{2}}-\frac{6 x_{t}^{2} \ln x_{t}}{\left(1-x_{t}\right)^{3}}\right],
\end{aligned}
$$

with $x_{t} \equiv m_{t}^{2} / M_{W}^{2}$. Here $\eta_{B}=0.84$ is the short-distance QCD correction calculated at NNLO [48], $f_{B_{q}}$ is the decay constant, and $\widehat{B}_{B_{q}}$ is the bag factor. The mass difference $\Delta M_{q}=2\left|M_{12}^{q}\right|$ is

$\Delta M_{q}=\Delta M_{q}^{\mathrm{SM}}\left|1+\frac{\left(g_{L}^{b q}\right)^{2}}{2 N C_{\mathrm{VLL}}^{\mathrm{SM}} M_{Z^{\prime}}^{2}}\right|$,

while the relevant weak phase $\phi_{q}$ is

$\phi_{q}=-2 \beta_{q}=\arg \left(M_{12}^{q}\right)$.

\subsection{Neutrino trident production}

Within the $Z^{\prime}$ models, the modification of the cross section $\sigma$ for neutrino trident production, $v_{\mu} N \rightarrow v_{\mu} N \mu^{+} \mu^{-}$may be parameterized as [49]

$$
\begin{aligned}
R_{\nu}=\frac{\sigma}{\sigma_{\mathrm{SM}}}= & \frac{1}{1+\left(1+4 s_{W}^{2}\right)^{2}}\left[\left(1+\frac{v^{2} g_{L}^{\mu \mu}\left(g_{L}^{\mu \mu}-g_{R}^{\mu \mu}\right)}{M_{Z^{\prime}}^{2}}\right)^{2}\right. \\
& \left.+\left(1+4 s_{W}^{2}+\frac{v^{2} g_{L}^{\mu \mu}\left(g_{L}^{\mu \mu}+g_{R}^{\mu \mu}\right)}{M_{Z^{\prime}}^{2}}\right)^{2}\right],
\end{aligned}
$$

where $v=246 \mathrm{GeV}$ and $s_{W}=\sin \theta_{W}$.

\section{Fit methodology}

We now determine favored values of the new physics couplings $g_{L}^{b s}, g_{L}^{b d}, g_{L}^{\mu \mu}$ and $g_{R}^{\mu \mu}$. We nominally take the mass of the $Z^{\prime}$ boson to be $M_{Z^{\prime}}=1 \mathrm{TeV}$. Note that since $M_{Z^{\prime}}$ only appears through the combination $g^{2} / M_{Z^{\prime}}^{2}$, the constraints on couplings can be appropriately scaled with the actual value of $M_{Z^{\prime}}$.

In $b \rightarrow s \mu^{+} \mu^{-}$decays, we consider the following observables: (i) $B_{S} \rightarrow \mu^{+} \mu^{-}$branching ratio [50-52], (ii) the updated value of $R_{K}$ by the LHCb collaboration [4], (iii) $R_{K^{*}}$ measured by LHCb [3] and its new Belle measurements, reported at Moriond'19 [53] (for Belle results, we use measurements in the bins $0.045 \mathrm{GeV}^{2}<q^{2}<1.1$ $\mathrm{GeV}^{2}, 1.1 \mathrm{GeV}^{2}<q^{2}<6.0 \mathrm{GeV}^{2}$, and $15.0 \mathrm{GeV}^{2}<q^{2}$ $<19.0 \mathrm{GeV}^{2}$, for $B^{0}$ as well as $B^{+}$decays), (iv) the differential branching ratios of $B_{d} \rightarrow K^{*} \mu^{+} \mu^{-}$[54-57], $B^{+} \rightarrow K^{*+} \mu^{+} \mu^{-}, B_{d} \rightarrow K \mu^{+} \mu^{-}, B^{+} \rightarrow K^{+} \mu^{+} \mu^{-}$ [55,58], and $B \rightarrow X_{s} \mu^{+} \mu^{-}$[59] in several $q^{2}$ bins, (v) various $C P$-conserving and $C P$-violating angular observables in $B_{d} \rightarrow K^{*} \mu^{+} \mu^{-}[10,55,57,60,61]$, (vi) the measurements of differential branching ratio and angular observables of $B_{s} \rightarrow \phi \mu^{+} \mu^{-}[8]$ in several $q^{2}$ bins.

While the ratios $R_{K}$ and $R_{K} *$ are theoretically clean, other observables are plagued by sizeable uncertainties mainly coming from form factors. For $B_{S} \rightarrow \phi$ and $B \rightarrow K$ decays, we use the most precise form factor predictions obtained in light cone sum rule (LCSR) [62,63], taking into account the correlations between the uncertainties of different form factors and at different $q^{2}$ values. The non-factorizable corrections are taken into account following the parameterization used in Ref. [63,64]. These are also compatible with the computations in Ref. [65].

All the observables in the $b \rightarrow s \mu^{+} \mu^{-}$sector put constraints on the combinations $g_{L}^{b s} g_{L}^{\mu \mu}$ and $g_{L}^{b s} g_{R}^{\mu \mu}$. For the fit related to $b \rightarrow s \mu^{+} \mu^{-}$, we closely follow the methodology of Ref. [13]. The $\chi^{2}$ function for all the $b \rightarrow s \mu^{+} \mu^{-}$ observables listed above is calculated as

$\chi_{b \rightarrow s \mu \mu}^{2}\left(C_{i}\right)=\left[\mathcal{O}_{\mathrm{th}}\left(C_{i}\right)-\mathcal{O}_{\exp }\right]^{T} \mathcal{C}^{-1}\left[\mathcal{O}_{\mathrm{th}}\left(C_{i}\right)-\mathcal{O}_{\exp }\right]$,

where $C_{i}=C_{9,10}^{b s, \mathrm{NP}}$. Here $\mathcal{O}_{\mathrm{th}}\left(C_{i}\right)$ are the theoretical predictions of $b \rightarrow s \mu^{+} \mu^{-}$observables calculated using flavio [64], and $\mathcal{O}_{\text {exp }}$ are the corresponding experimental measure- 
ments. The total covariance matrix $\mathcal{C}$ is obtained by adding the individual theoretical and experimental covariance matrices. In order to get the theoretical uncertainties, including the correlations among them, all input parameters such as the form factors, bag parameters, masses of particles, decay constants etc. are varied assuming a Gaussian distribution, following the same methodology as used in flavio [64]. For the experimental covariance, we take into account the correlations among the angular observables in $B \rightarrow K^{(*)} \mu^{+} \mu^{-}$ [10] and $B_{s} \rightarrow \phi \mu^{+} \mu^{-}$[8]. For the other observables, we add the statistical and systematic errors in quadrature. Wherever the errors are asymmetric, we use the conservative approach of using the larger error on both sides of the central value.

We now turn to $B_{q}-\bar{B}_{q}$ mixing. Here we consider constraints from $\Delta M_{d}, \Delta M_{s}$, and the two CP-violating phases. Using $f_{B_{d}} \sqrt{\widehat{B}_{B_{d}}}=(225 \pm 9) \mathrm{MeV}$ [66], along with other input parameters from Ref. [67], Eq. (8) gives $\Delta M_{d}^{\mathrm{SM}}=(0.547 \pm 0.046) \mathrm{ps}^{-1}$. With $\Delta M_{d}^{\exp }=(0.5065 \pm$ $0.0019) \mathrm{ps}^{-1}$ [68], the contribution of $\Delta M_{d}$ to $\chi^{2}$ is

$$
\chi_{\Delta M_{d}}^{2}=\left(\frac{\Delta M_{d}-\Delta M_{d}^{\text {exp }, \mathrm{m}}}{\sigma_{\Delta M_{d}}}\right)^{2},
$$

where we denote the experimental mean value of an observable $X$ by $X^{\text {exp,m}}$, and the uncertainty in the observable by $\sigma_{X}$. In order to obtain $\sigma_{X}$, we add the experimental and theoretical uncertainties in quadrature. Here, $\sigma_{\Delta M_{d}}$ is dominated by the theoretical uncertainty.

In order to minimize the impact of theoretical uncertainties, we use $\Delta M_{S}$ constraints through the ratio $M_{R}=$ $\Delta M_{d} / \Delta M_{s}$. In the SM,

$M_{R}^{\mathrm{SM}}=\left|\frac{V_{t d}}{V_{t s}}\right|^{2} \frac{1}{\xi^{2}} \frac{M_{B_{d}}}{M_{B_{s}}}$,

where $\xi=\frac{f_{B_{d}}^{2} \widehat{B}_{B_{d}}}{f_{B_{s}}^{2} \widehat{B}_{B_{s}}}$. Using $\xi=1.2014_{-0.0072}^{+0.0065}$ [69] and $\left|V_{t d} / V_{t s}\right|=0.2088_{-0.0030}^{+0.0016}$ [70], we obtain $M_{R}^{S M}=0.0297 \pm$ 0.0009 , where we have added the errors in quadrature. Wherever there are asymmetric errors, we take a conservative approach and use the larger of the errors on two sides. The value of $M_{R}^{\exp }=0.0285 \pm 0.0001$ [68], so the contribution to $\chi^{2}$ due to this ratio is

$\chi_{M_{R}}^{2}=\left(\frac{M_{R}-M_{R}^{\text {exp }, \mathrm{m}}}{\sigma_{M_{R}}}\right)^{2}$.

The observables $\Delta M_{d}$ and $M_{R}$ constrain $\left|g_{L}^{b s}\right|$ and $\left|g_{L}^{b d}\right|$.
The $C P$-violating constraints from $J / \psi \phi$ and $J / \psi K_{S}$ decays contribute to the $\chi^{2}$ as

$$
\begin{aligned}
\chi_{J / \psi \phi}^{2} & =\left(\frac{S_{J / \psi \phi}-S_{J / \psi \phi}^{\exp , \mathrm{m}}}{\sigma_{S_{J / \psi} \phi}}\right)^{2}, \\
\chi_{J / \psi K_{S}}^{2} & =\left(\frac{S_{J / \psi K_{S}}-S_{J / \psi K_{S}}^{\exp , \mathrm{m}}}{\sigma_{S_{J / \psi K_{S}}}}\right)^{2},
\end{aligned}
$$

where $S_{J / \psi \phi}=-\operatorname{Im}\left[M_{12}^{s}\right] /\left|M_{12}^{s}\right|$ and $S_{J / \psi K_{S}}=\operatorname{Im}\left[M_{12}^{d}\right] /\left|M_{12}^{d}\right|$. Here we have taken the measurements to be $S_{J / \psi \phi}^{\exp }=$ $0.02 \pm 0.03$ and $S_{J / \psi K_{S}}^{\exp }=0.69 \pm 0.02$ [67].

For the constraints from neutrino trident production, we use the quantity $R_{v}=\sigma / \sigma_{\mathrm{SM}}$, whose theoretical expression is given in Eq. (10). We have taken $R_{\nu}^{\exp } \equiv 0.82 \pm 0.28$ [71,72]. The contribution to the total $\chi^{2}$ is

$\chi_{\text {trident }}^{2}=\left(\frac{R_{\nu}-R_{\nu}^{\text {exp, } \mathrm{m}}}{\sigma_{R_{v}}}\right)^{2}$.

This observable constraints $g_{L}^{\mu \mu}$ and $g_{R}^{\mu \mu}$.

The $b \rightarrow d \mu^{+} \mu^{-}$decays are CKM-suppressed as compared to $b \rightarrow s \mu^{+} \mu^{-}$. In our analysis, we include constraints from the branching ratios of $B^{+} \rightarrow \pi^{+} \mu^{+} \mu^{-}$and $B_{d} \rightarrow \mu^{+} \mu^{-}$decays. We do not include the measurements of observables in $B_{s} \rightarrow \bar{K}^{*} \mu^{+} \mu^{-}$decay in our fit, since we are interested in obtaining predictions for these.

The theoretical expression for $\mathcal{B}\left(B^{+} \rightarrow \pi^{+} \mu^{+} \mu^{-}\right)$in the $Z^{\prime}$ model can be obtained from Ref. [73], by adding the $\mathrm{NP}$ contribution as given in Eq. (5). The contribution to $\chi^{2}$ from this decay is

$\chi_{B^{+} \rightarrow \pi \mu \mu}^{2}=\left(\frac{\mathscr{B}\left(B^{+} \rightarrow \pi \mu \mu\right)-\mathcal{B}\left(B^{+} \rightarrow \pi \mu \mu\right)^{\mathrm{exp}, \mathrm{m}}}{\sigma_{\mathcal{B}\left(B^{+} \rightarrow \pi \mu \mu\right)}}\right)^{2}$,

where $\mathcal{B}\left(B^{+} \rightarrow \pi \mu \mu\right)^{\exp }=(1.83 \pm 0.24) \times 10^{-8}$ [41]. Following Ref. [73], a theoretical error of $15 \%$ is included due to uncertainties in the $B \rightarrow \pi$ form factors [74]. by

The branching ratio of $B_{d} \rightarrow \mu^{+} \mu^{-}$in our model is given

$$
\begin{aligned}
\mathcal{B}\left(B_{d} \rightarrow \mu^{+} \mu^{-}\right) & =\frac{G_{F}^{2} \alpha^{2} M_{B_{d}} m_{\mu}^{2} f_{B_{d}}^{2} \tau_{B_{d}}}{16 \pi^{3}}\left|V_{t d} V_{t b}^{*}\right|^{2} \\
& \times \sqrt{1-\frac{4 m_{\mu}^{2}}{M_{B_{d}}^{2}}}\left|C_{10}^{b d, \mathrm{SM}}+C_{10}^{b d, N P}\right|^{2},
\end{aligned}
$$

and the contribution to $\chi^{2}$ is

$$
\chi_{B_{d} \rightarrow \mu \mu}^{2}=\left(\frac{\mathcal{B}\left(B_{d} \rightarrow \mu \mu\right)-\mathcal{B}\left(B_{d} \rightarrow \mu \mu\right)^{\text {exp }, \mathrm{m}}}{\sigma_{\mathcal{B}\left(B_{d} \rightarrow \mu \mu\right)}}\right)^{2} .
$$




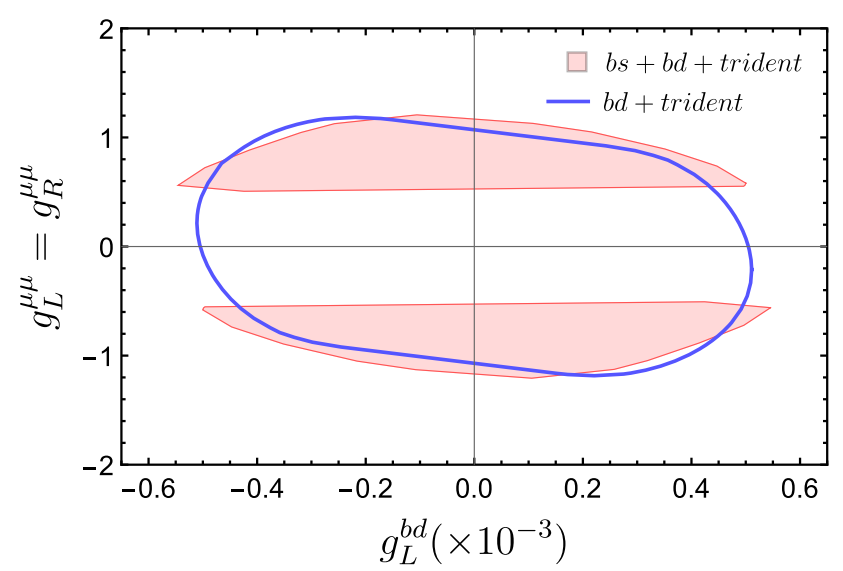

Fig. 1 The $\left(g_{L}^{b d}, g_{L}^{\mu \mu}\right)$ parameter space corresponding to a $Z^{\prime}$ model with $g_{L}^{\mu \mu}=g_{R}^{\mu \mu}$ (i.e. $C_{10}^{b d, N P}=0$ ), for $M_{Z^{\prime}}=1 \mathrm{TeV}$. The blue curve is the boundary of the $1 \sigma$-favored region due to constraints from measurements in $b \rightarrow d$ sector and neutrino trident production. The pink shaded region represents the $1 \sigma$-favored parameter space after including additional constraints from $b \rightarrow s \mu^{+} \mu^{-}$data and $B_{s}-\bar{B}_{s}$ mixing

We have used $\mathcal{B}\left(B_{d} \rightarrow \mu^{+} \mu^{-}\right)^{\exp }=(3.9 \pm 1.6) \times 10^{-10}$ [68], $f_{B_{d}}=(190 \pm 1.3) \mathrm{MeV}$ [69], and other inputs from [67].

Finally, combining all the above constraints, we obtain

$$
\begin{aligned}
\chi_{\text {total }}^{2}= & \chi_{b \rightarrow s \mu \mu}^{2}+\chi_{\Delta M_{d}}^{2}+\chi_{M_{R}}^{2}+\chi_{J / \Psi \phi}^{2}+\chi_{J / \Psi K_{S}}^{2}+\chi_{\text {trident }}^{2} \\
& +\chi_{B^{+} \rightarrow \pi \mu \mu}^{2}+\chi_{B_{d} \rightarrow \mu \mu}^{2} .
\end{aligned}
$$

In addition to the above constraints, there would be constraints coming from $b \rightarrow s v \bar{v}$ and charm sector. However, at present, we only have upper limits on $b \rightarrow s v \bar{v}$ and $c \rightarrow u \mu^{+} \mu^{-}$decays. Further, in the $D^{0}-\bar{D}^{0}$ mixing, we expect a large fraction of unknown long-distance contributions. Therefore these measurements cannot be included in as clean a manner as the ones we have considered above. Instead, in the appendices A and B, we determine the allowed regions due to these constraints taken separately and compare with those obtained from our fit. We find that the constraints from these additional channels are much weaker, and will not affect our results.

In the next section, we present our fit results, along with predictions of several observables in $B_{S} \rightarrow \bar{K}^{*} \mu^{+} \mu^{-}$decay.

\section{Fit results and predictions}

In a model-independent analysis, there have been attempts to put limits on the new physics couplings for $b \rightarrow d \ell \ell$ decays [75], however it is difficult as there are only a few measurements in this sector. Within the context of a $Z^{\prime}$ model, one can obtain meaningful constraints using correlated $b \rightarrow s$ and $b \rightarrow d$ processes. This can be seen from Fig. 1, which depicts the allowed $\left(g_{L}^{b d}, g_{L}^{\mu \mu}=g_{R}^{\mu \mu}\right)$ parameter space corresponding to a $Z^{\prime}$ model which generates the $1 \mathrm{D}$ scenario $C_{10}^{b d, \mathrm{NP}}=0$. The elliptical region represents the $1 \sigma$-favored parameter space with constraints only from $b \rightarrow d$ sector, i.e., branching ratios of $B^{+} \rightarrow \pi^{+} \mu^{+} \mu^{-}$and $B_{d} \rightarrow \mu^{+} \mu^{-}$ decays, $B_{d}-\overline{B_{d}}$ mixing, and neutrino trident production. The two shaded regions represent the $1 \sigma$-favored parameter space obtained by including additional constraints from all relevant measurements related to $b \rightarrow s \mu^{+} \mu^{-}$decays and $B_{S}-\bar{B}_{s}$ mixing. It can be seen that the allowed range of NP couplings, in particular $g_{L, R}^{\mu \mu}$, reduces considerably after including constraints from the $b \rightarrow s$ sector. Therefore, it is worth studying implications of several measurements in the $b \rightarrow s$ sector on the observables in $b \rightarrow d \mu^{+} \mu^{-}$decays.

Performing a fit to the relevant observables in $b \rightarrow s$ and $b \rightarrow d$ sectors, we determine the $1 \sigma$-favored parameter space of the couplings $g_{L}^{b d}, g_{L}^{b s}, g_{L}^{\mu \mu}$ and $g_{R}^{\mu \mu}$, considering $g_{L}^{b s}$ and $g_{L}^{b d}$ to be (i) real, (ii) complex. These can be used to find constraints on the NP Wilson coefficients $\left(C_{9}^{b d, \mathrm{NP}}, C_{10}^{b d, \mathrm{NP}}\right)$, and to put limits on the allowed NP in the following observables in $B_{s} \rightarrow \bar{K}^{*} \mu^{+} \mu^{-}$decay: differential branching ratio, the LFUV ratio $R_{K^{*}}^{(s)}$, muon forward-backward asymmetry $A_{F B}$, longitudinal polarization fraction $F_{L}$, and direct $C P$ asymmetry $A_{C P}$.

The matrix element for the decay amplitude of $B_{s} \rightarrow$ $\bar{K}^{*} \mu^{+} \mu^{-}$can be written as

$$
\begin{aligned}
\mathcal{M}= & \frac{G_{F} \alpha}{\sqrt{2} \pi} V_{t b} V_{t d}^{*}\left\{\left[C_{9}^{b d}\left\langle\bar{K}^{*}\left|\bar{d} \gamma^{\mu} P_{L} b\right| B_{s}\right\rangle\right.\right. \\
& \left.-\frac{2 m_{b}}{q^{2}} C_{7}^{b d}\left\langle\bar{K}^{*}\left|\bar{d} i \sigma^{\mu \nu} q_{\nu} P_{R} b\right| B_{s}\right\rangle\right]\left(\bar{\mu} \gamma_{\mu} \mu\right) \\
& \left.+C_{10}^{b d}\left\langle\bar{K}^{*}\left|\bar{d} \gamma^{\mu} P_{L} b\right| B_{S}\right\rangle\left(\bar{\mu} \gamma_{\mu} \gamma_{5} \mu\right)\right\}
\end{aligned}
$$

where $C_{9}^{b d, \mathrm{SM}}$ and $C_{10}^{b d, \mathrm{SM}}$ are taken from Ref. [76] and Ref. [77] respectively. The matrix elements appearing in Eq. (21) have been calculated using form factors obtained by a combined fit to lattice calculations and QCD sum rules on the light cone [63]. We also include the non-factorizable corrections due to soft gluon emission and charmonium resonance, which have been computed for $B_{d} \rightarrow K^{*} \ell \ell[65,78]$, and parameterized as corrections to $C_{9}^{\mathrm{SM}}$. These effects are assumed to be roughly the same for $B_{s} \rightarrow \bar{K}^{*} \ell \ell$ due to flavor symmetry [79].

The decay $B_{s} \rightarrow \bar{K}^{*} \mu^{+} \mu^{-}$may be described in terms of the fourfold distribution as [79]

$$
\begin{aligned}
\frac{d \Gamma}{d q^{2}} & =\int_{-1}^{+1} d \cos \theta_{l} d \cos \theta_{V} \int_{0}^{\pi} d \phi \frac{d^{4} \Gamma}{d q^{2} d \cos \theta_{V} d \cos \theta_{l} d \phi} \\
& =\frac{1}{4}\left(3 I_{1}^{c}+6 I_{1}^{s}-I_{2}^{c}-2 I_{2}^{s}\right),
\end{aligned}
$$



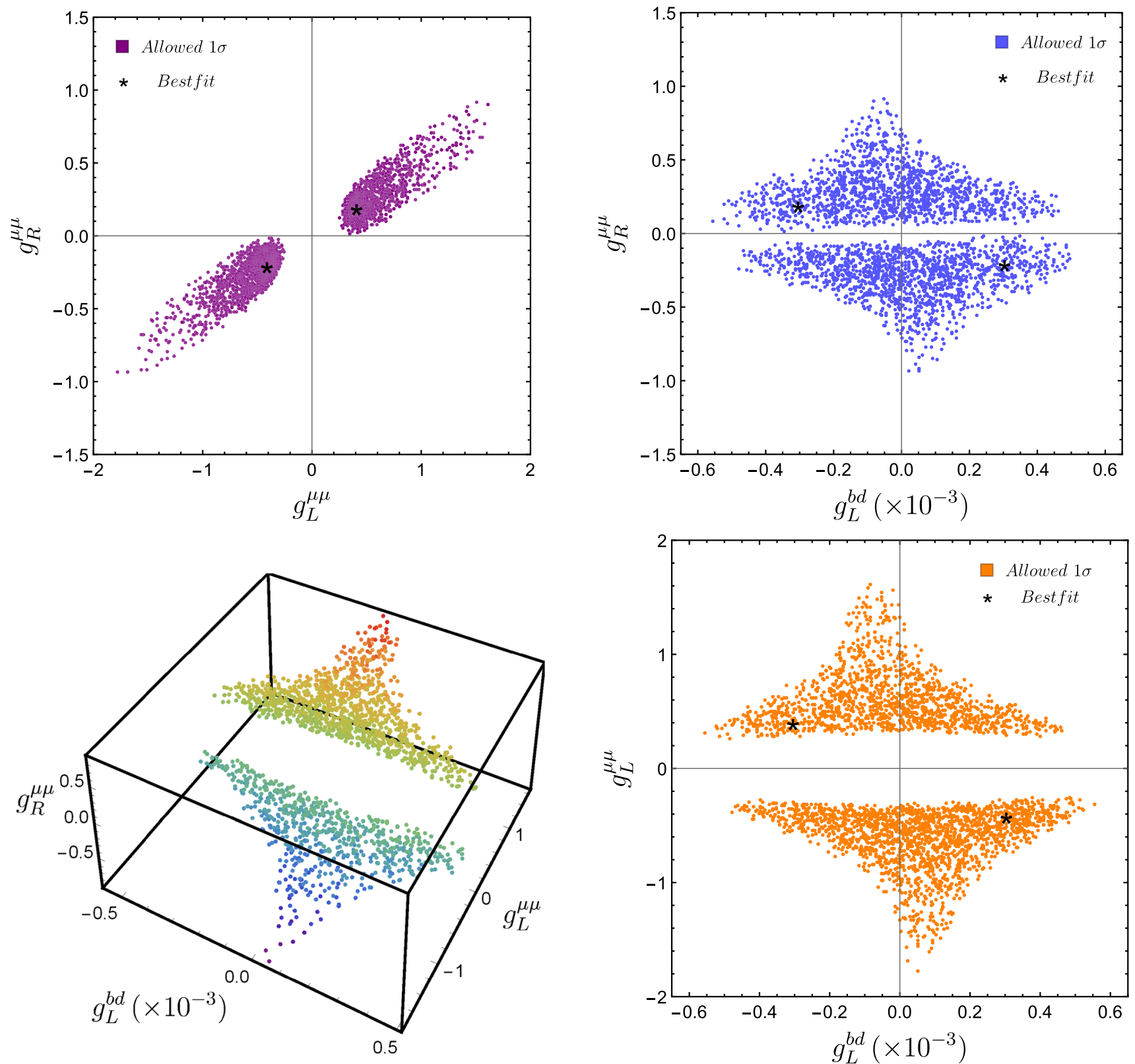

Fig. 2 The $1 \sigma$-favored $\left(g_{L}^{b d}, g_{L}^{\mu \mu}, g_{R}^{\mu \mu}\right)$ parameter space for a $Z^{\prime}$ model with real couplings, for $M_{Z^{\prime}}=1$ TeV. The colors red to blue in the bottom left 3D parameter space correspond to decreasing values of $g_{R}^{\mu \mu}$

Table 1 Values of NP Wilson coefficients for benchmark scenarios NP1, NP2 corresponding to a $Z^{\prime}$ model with real couplings, and scenarios NP3, NP4 with complex couplings. The first two benchmark scenarios NP1 and NP2 for real $Z^{\prime}$ couplings, correspond to a maxi- mum deviation from the SM predictions of the observables considered. The last two scenarios, for complex couplings, are the $1 \sigma$-favoured ones with a near-maximum value of $\operatorname{Im}\left[C_{9}^{b d, N P}\right]$ and a near-minimum value of $\operatorname{Re}\left[C_{10}^{b d, \mathrm{NP}}\right]$

\begin{tabular}{lllll}
\hline Scenario & $\mathrm{NP} 1$ & $\mathrm{NP} 2$ & $\mathrm{NP} 3$ & $\mathrm{NP} 4$ \\
\hline$C_{9}^{b d, \mathrm{NP}}$ & +0.98 & -0.80 & $-1.4+4.9 i$ & $-0.6+0.8 i$ \\
$C_{10}^{b d, \mathrm{NP}}$ & -0.17 & +0.19 & $+0.7-2.3 i$ & $+0.2-0.2 i$ \\
\hline
\end{tabular}



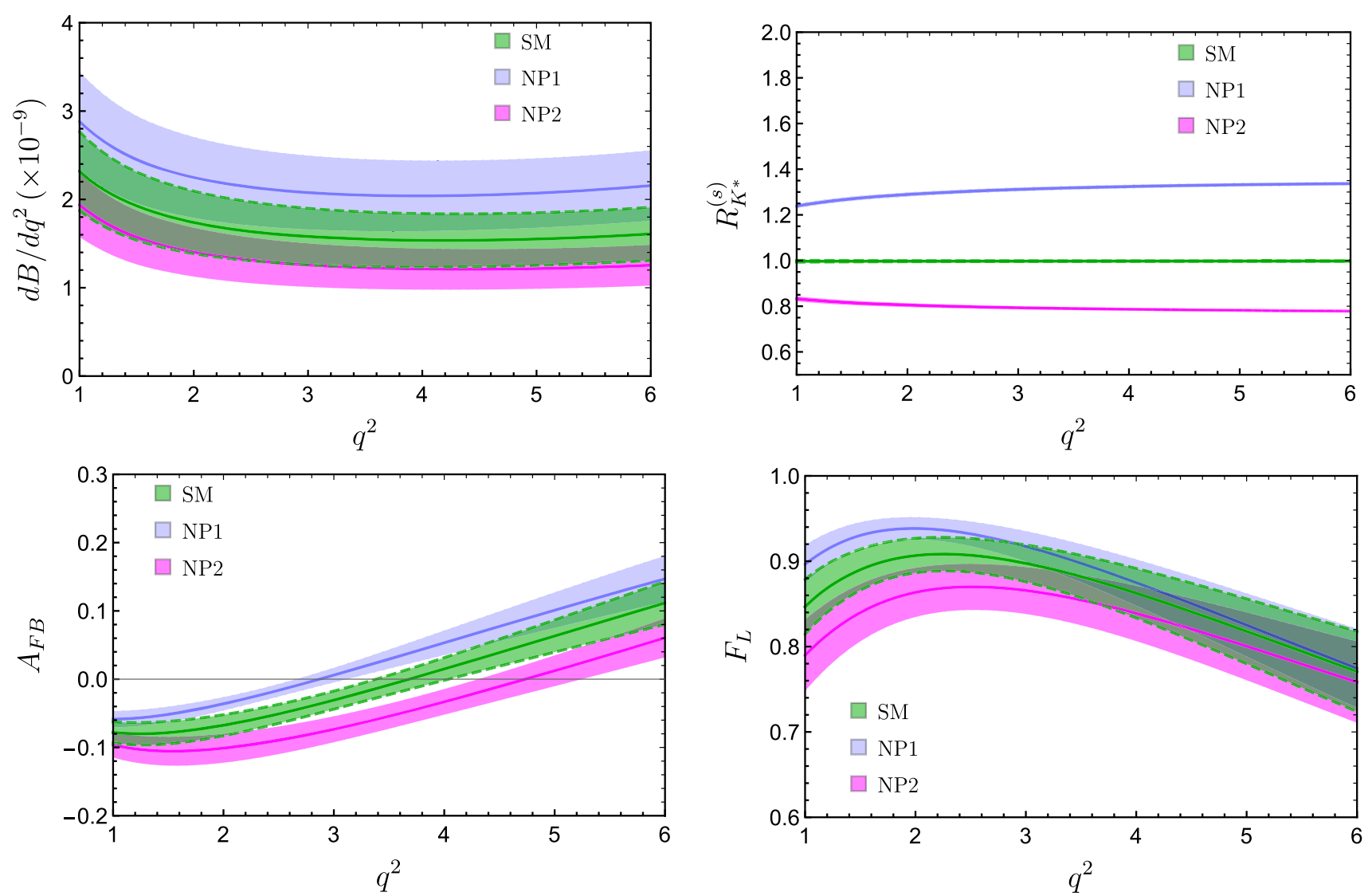

Fig. 3 The predictions for observables in $B_{s} \rightarrow \bar{K}^{*} \mu^{+} \mu^{-}$decay, with real $Z^{\prime}$ couplings, for SM and the benchmark scenarios NP1 and NP2 as in Table 1

where $q^{2}$ is the lepton invariant mass, $\theta_{V}$ and $\theta_{l}$ are the polar angles, and $\phi$ is the angle between the dimuon plane and $K^{*}$ decay plane. The relevant observables can be obtained from the four-fold distribution as

$$
\begin{aligned}
\frac{d B}{d q^{2}} & =\tau_{B_{s}} \frac{d \Gamma}{d q^{2}}, \\
R_{K^{*}}^{(s)}\left(q^{2}\right) & =\frac{d \Gamma\left(B_{s} \rightarrow \bar{K}^{*} \mu^{+} \mu^{-}\right) / d q^{2}}{d \Gamma\left(B_{s} \rightarrow \bar{K}^{*} e^{+} e^{-}\right) / d q^{2}}, \\
A_{F B}\left(q^{2}\right) & =\frac{1}{d \Gamma / d q^{2}}\left[\int_{-1}^{0}-\int_{0}^{1}\right] d \cos \theta_{l} \frac{d^{4} \Gamma}{d q^{2} d \cos \theta_{l}} \\
& =\frac{-3 I_{6}^{s}}{3 I_{1}^{c}+6 I_{1}^{s}-I_{2}^{c}-2 I_{2}^{s}}, \\
F_{L}\left(q^{2}\right) & =\frac{3 I_{1}^{c}-I_{2}^{c}}{3 I_{1}^{c}+6 I_{1}^{s}-I_{2}^{c}-2 I_{2}^{s}}, \\
A_{C P}\left(q^{2}\right) & =\frac{d B / d q^{2}-d \bar{B} / d q^{2}}{d B / d q^{2}+d \bar{B} / d q^{2}},
\end{aligned}
$$

where the functions $I_{i}$ can be expressed in terms of the transversity amplitudes [80]. Here $\bar{B}$ corresponds to the decay mode $\bar{B}_{s} \rightarrow K^{*} \mu^{+} \mu^{-}$.
We present our results for the above observables at four benchmark NP scenarios as given in Table 1.

\subsection{Real couplings}

In order to quantify how well the $Z^{\prime}$ model is able to account for all data in the $b \rightarrow s$ and $b \rightarrow d$ sectors, we define $\Delta \chi^{2}=\chi_{\mathrm{SM}}^{2}-\chi_{\mathrm{NP}}^{2}$, where the minimum $\chi^{2}$ in the SM, and in the presence of NP $Z^{\prime}$ couplings, is denoted by $\chi_{\mathrm{SM}}^{2}$ and $\chi_{\mathrm{NP}}^{2}$, respectively. For the case of real couplings, we find the best fit values to be $g_{L}^{b d}= \pm 0.3 \times 10^{-3}, g_{L}^{\mu \mu}=\mp 0.4$, and $g_{R}^{\mu \mu}=$ $\mp 0.2$. The value of $\chi_{\mathrm{SM}}^{2} \approx 221$ and $\Delta \chi^{2} \approx 41$. The value of $\chi_{\mathrm{SM}}^{2}=221$ corresponds to $g_{L}^{b s}=g_{L}^{b d}=g_{L}^{\mu \mu}=g_{R}^{\mu \mu}=0$. Since allowing all these NP couplings to be non-zero can decrease the $\chi^{2}$ to $\chi_{\mathrm{NP},(s, d)}^{2} \approx 181$, the SM point may be said to be highly disfavoured. However, even if we restrict $g_{L}^{b d}=0$, the freedom allowed in the other NP couplings can still allow $\chi_{\mathrm{NP}, s}^{2} \approx 181$. Thus, the improvement over the SM, $\Delta \chi^{2} \approx 41$ is mainly due to the presence of non-zero $g_{L}^{b s}$ and the muon couplings, which help explain the anomalies in the $b \rightarrow$ sll sector.

The $1 \sigma$-favored parameter space of the couplings $\left(g_{L}^{b d}\right.$, $\left.g_{L}^{\mu \mu}, g_{R}^{\mu \mu}\right)$ is shown in Fig. 2. It can be seen from $\left(g_{L}^{b d}, g_{L}^{\mu \mu}\right)$ 

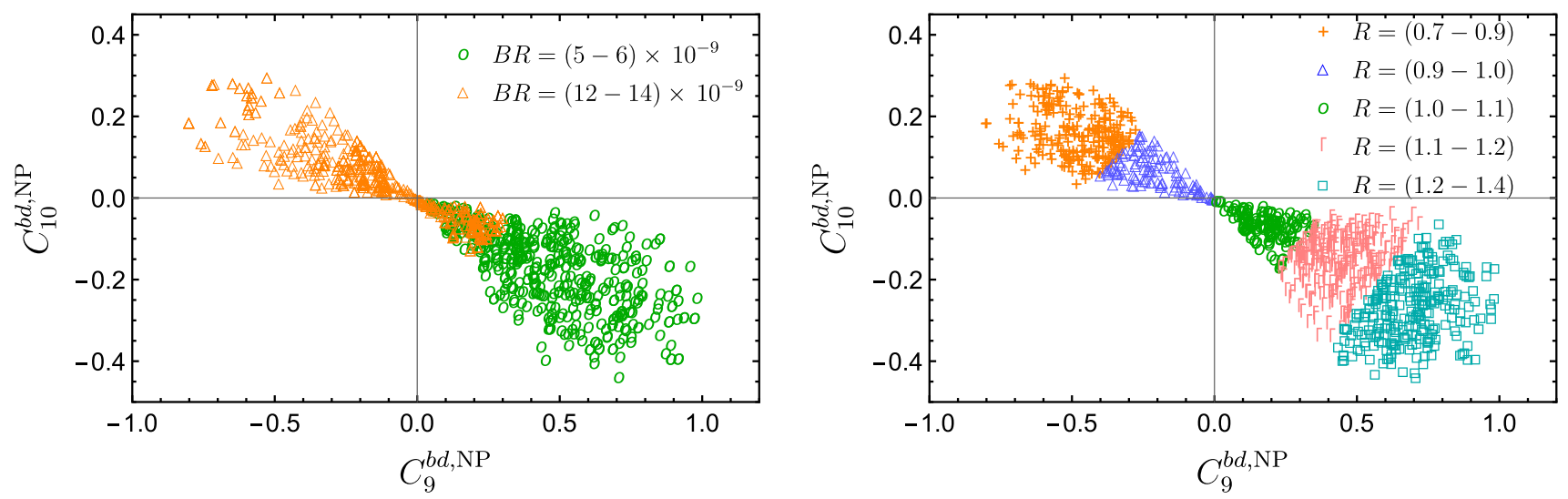

Fig. 4 Integrated values of the branching ratio of $B_{s} \rightarrow \bar{K}^{*} \mu^{+} \mu^{-}$(left panel) and the LFUV ratio $R_{K^{*}}^{(s)}$ (right panel) in the $1 \sigma$-favored parameter space of $\left(C_{9}^{b d, \mathrm{NP}}, C_{10}^{b d, \mathrm{NP}}\right)$

and $\left(g_{L}^{b d}, g_{R}^{\mu \mu}\right)$ planes that, while $g_{R}^{\mu \mu}=0$ is barely disfavored within $1 \sigma$, a rather wide strip $\left|g_{L}^{\mu \mu}\right| \leq 0.25$ lies beyond the $1 \sigma$-favored region. This is because the anomalies in $b \rightarrow s \mu \mu$ decays need a non-zero value of $C_{9}^{b s, \mathrm{NP}}$, which in turn require a non-zero value of $g_{L}^{\mu \mu}$ or $g_{R}^{\mu \mu}$. Furthermore, the scenario $C_{9}^{b s, \mathrm{NP}}=-C_{10}^{b s, \mathrm{NP}}$ [13], which provides a good fit, favors $g_{R}^{\mu \mu}=0$, thus requiring $g_{L}^{\mu \mu}$ to be away from zero. Note that the results in the $\left(g_{L}^{\mu \mu}, g_{R}^{\mu \mu}\right)$ plane indicate the class of favored solutions that lie along $g_{L}^{\mu \mu}=g_{R}^{\mu \mu}$, corresponding to $C_{10}^{b s, \mathrm{NP}} \approx 0 \approx C_{10}^{b d, \mathrm{NP}}$.

\subsubsection{Predictions for $d B / d q^{2}, R_{K^{*}}^{(s)}\left(q^{2}\right), A_{F B}\left(q^{2}\right)$ and $F_{L}\left(q^{2}\right)$}

The top left panel of Fig. 3 shows predictions for the differential branching ratio corresponding to real $Z^{\prime}$ couplings, for the SM as well as two benchmark scenarios NP1 and NP2 from Table. 1. These scenarios roughly correspond to the maximum deviation on either side from the SM predictions in the $1 \sigma$ favored NP parameter space. The maximum enhancement (suppression) in the differential branching ratio corresponds roughly to a maximum positive (negative) value of $C_{9}^{b d, \mathrm{NP}}$. It can be seen from the figure that only a marginal enhancement or suppression over the SM value is possible in the differential branching ratio. A clean distinction among the predictions of different scenarios is difficult owing to the large uncertainties (about 20\%) arising from the formfactors.

A measurement of the LFUV ratio $R_{K^{*}}^{(s)}\left(q^{2}\right)$ in a few $q^{2}$ bins would be possible with the LHCb upgrade-II data set [45]. The predictions for this quantity in the benchmark scenarios NP1 and NP2 are shown in the top right panel of Fig. 3. In the SM, $R_{K^{*}}^{(s)}\left(q^{2}\right)$ is unity in the entire low $-q^{2}$ region, while an enhancement up to 1.3 and a suppression up to 0.8 is allowed. The maximum enhancement (suppres- sion) roughly corresponds to the maximum positive (negative) value of $C_{9}^{b d, \mathrm{NP}}$.

Within the SM, the forward-backward asymmetry $A_{F B}\left(q^{2}\right)$ is predicted to vanish around $q^{2} \approx 3.5 \mathrm{GeV}^{2}$, and the zerocrossing is from negative to positive, as can be seen from the bottom left panel in Fig. 3. The maximum value of $A_{F B}\left(q^{2}\right)$ in the $\mathrm{SM}$ is $\approx 10 \%$. The positive (negative) value of $C_{9}^{b d, \mathrm{NP}}$ also shifts the zero-crossing towards lower (higher) $q^{2}$ value. The integrated value of $A_{F B}$ over $q^{2}=(1-6) \mathrm{GeV}^{2}$ bin is $(-0.6 \pm 1) \%$ within the SM . The predictions for integrated $A_{F B}$ for the benchmark scenarios NP1 and NP2 are $(3.1 \pm 1.4) \%$ and $(-5 \pm 1.7) \%$, respectively.

The predictions for longitudinal polarization fraction $F_{L}\left(q^{2}\right)$ are shown in the bottom right panel of Fig. 3. Within the SM, the peak value of $F_{L}\left(q^{2}\right)$ is $\approx 0.9$ around $q^{2} \approx 1.8$ $\mathrm{GeV}^{2}$. The shape of $F_{L}\left(q^{2}\right)$ does not change with NP and only a marginal deviation from SM is allowed for the benchmark NP scenarios considered here.

Thus, in the case of real couplings, $R_{K^{*}}^{(s)}\left(q^{2}\right)$ is useful to distinguish the predictions of the two benchmark NP scenarios from the SM expectation, while the predictions for the differential branching ratio, $A_{F B}\left(q^{2}\right)$, and $F_{L}\left(q^{2}\right)$ may not have distinct NP signatures, owing to the large form factor uncertainties.

\subsubsection{Integrated branching ratio and $R_{K^{*}}^{(s)}$ in the low- $q^{2}$ region}

The results obtained for integrated $d B / d q^{2}$ and $R_{K^{*}}^{(s)}\left(q^{2}\right)$ over the $q^{2}=(1-6) \mathrm{GeV}^{2}$ bin are presented in Fig. 4. These results are depicted in the $\left(C_{9}^{b d, \mathrm{NP}}, C_{10}^{b d, \mathrm{NP}}\right)$ plane, with different colors and symbols indicating the values of integrated branching ratio (left panel) and integrated $R_{K^{*}}^{(s)}$ (right panel). At each $1 \sigma$-favored value of $\left(C_{9}^{b d, \mathrm{NP}}, C_{10}^{b d, \mathrm{NP}}\right)$, we vary the values of form factor parameters within their $1 \sigma$ range [63] with a Gaussian distribution of uncertainties. 

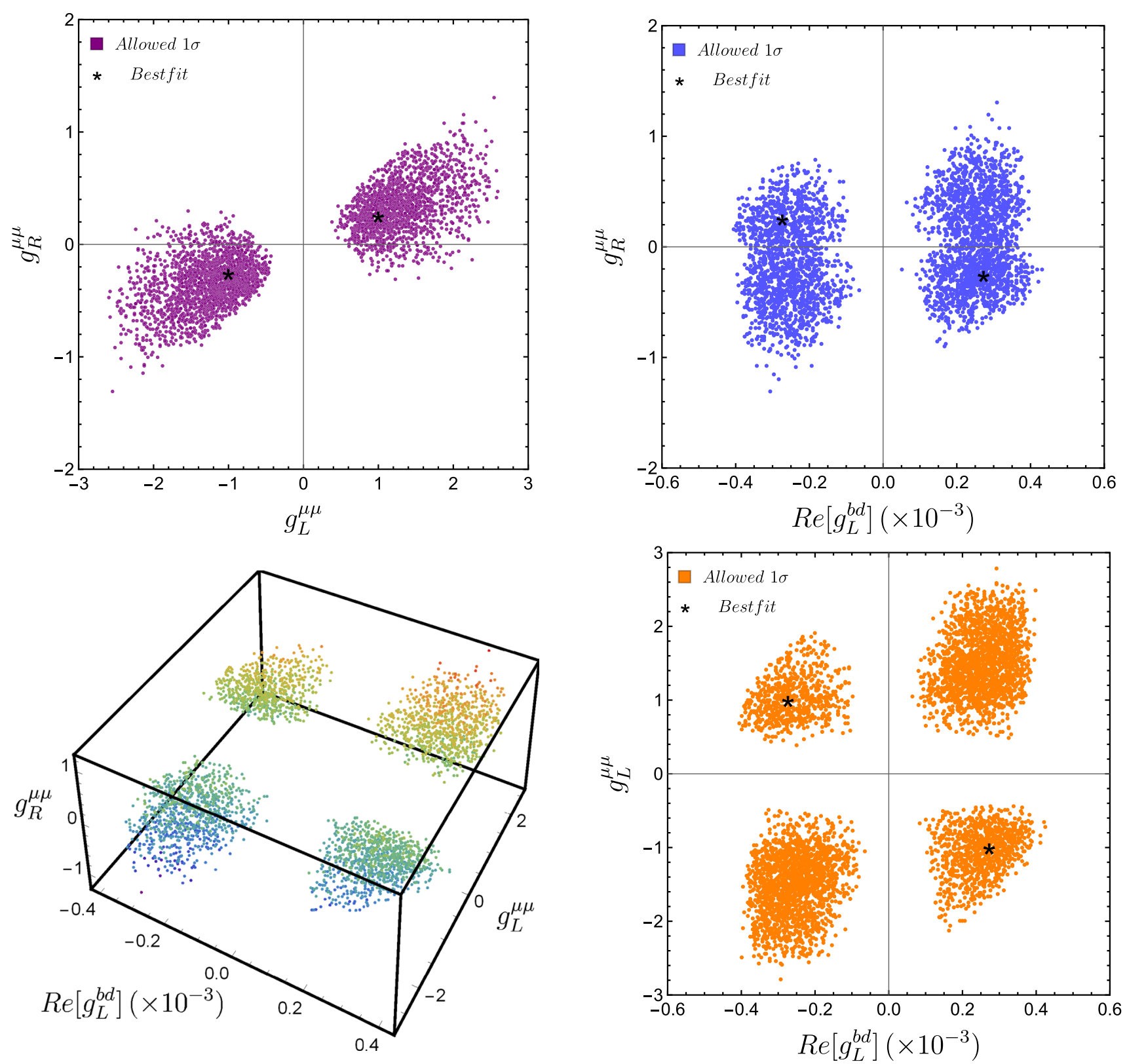

Fig. 5 The $1 \sigma$-favored $\left(\operatorname{Re}\left[g_{L}^{b d}\right], g_{L}^{\mu \mu}, g_{R}^{\mu \mu}\right)$ parameter space for a $Z^{\prime}$ model with complex couplings, for $M_{Z^{\prime}}=1 \mathrm{TeV}$. The colors red to blue in the bottom left 3D parameter space correspond to decreasing values of $g_{R}^{\mu \mu}$

In the case of integrated branching ratio, the errors due to form factors are about $20 \%$. Due to such large errors, even by considering branching ratio values as different as (5-6) $\times 10^{-9}$ and (12-14) $\times 10^{-9}$, we find a significant overlap in the $\left(C_{9}^{b d, \mathrm{NP}}, C_{10}^{b d, \mathrm{NP}}\right)$ plane. Hence, a measurement of integrated branching ratio may not be very helpful to put limits on the allowed values of the NP couplings.

In the case of $R_{K^{*}}^{(s)}$, the uncertainties due to form factors cancel in the ratio. The lack of overlap between the regions of integrated $R_{K^{*}}^{(s)}$ values in the range (0.7-1.4) indicates that a future measurement of integrated $R_{K^{*}}^{(s)}$ with an accuracy of $\sim 10 \%$ in this decay mode would make it possible to identify the ranges of $\left(C_{9}^{b d, \mathrm{NP}}, C_{10}^{b d, \mathrm{NP}}\right)$ more precisely. Even with a preliminary measurement, an enhancement in the value of $R_{K^{*}}^{(s)}$ above unity would indicate a positive value of $C_{9}^{b d, \mathrm{NP}}$ and a negative value of $C_{10}^{b d, \mathrm{NP}}$, while a suppression would imply a negative $C_{9}^{b d, \mathrm{NP}}$ and positive $C_{10}^{b d, \mathrm{NP}}$. This feature may be understood from the approximate analytic form of the LFUV ratio, $R_{K^{*}}^{(s)} \propto\left(\operatorname{Re}\left[C_{9}^{b q, \mathrm{NP}}\right]-\operatorname{Re}\left[C_{10}^{b q, \mathrm{NP}}\right]\right)[81]$. 

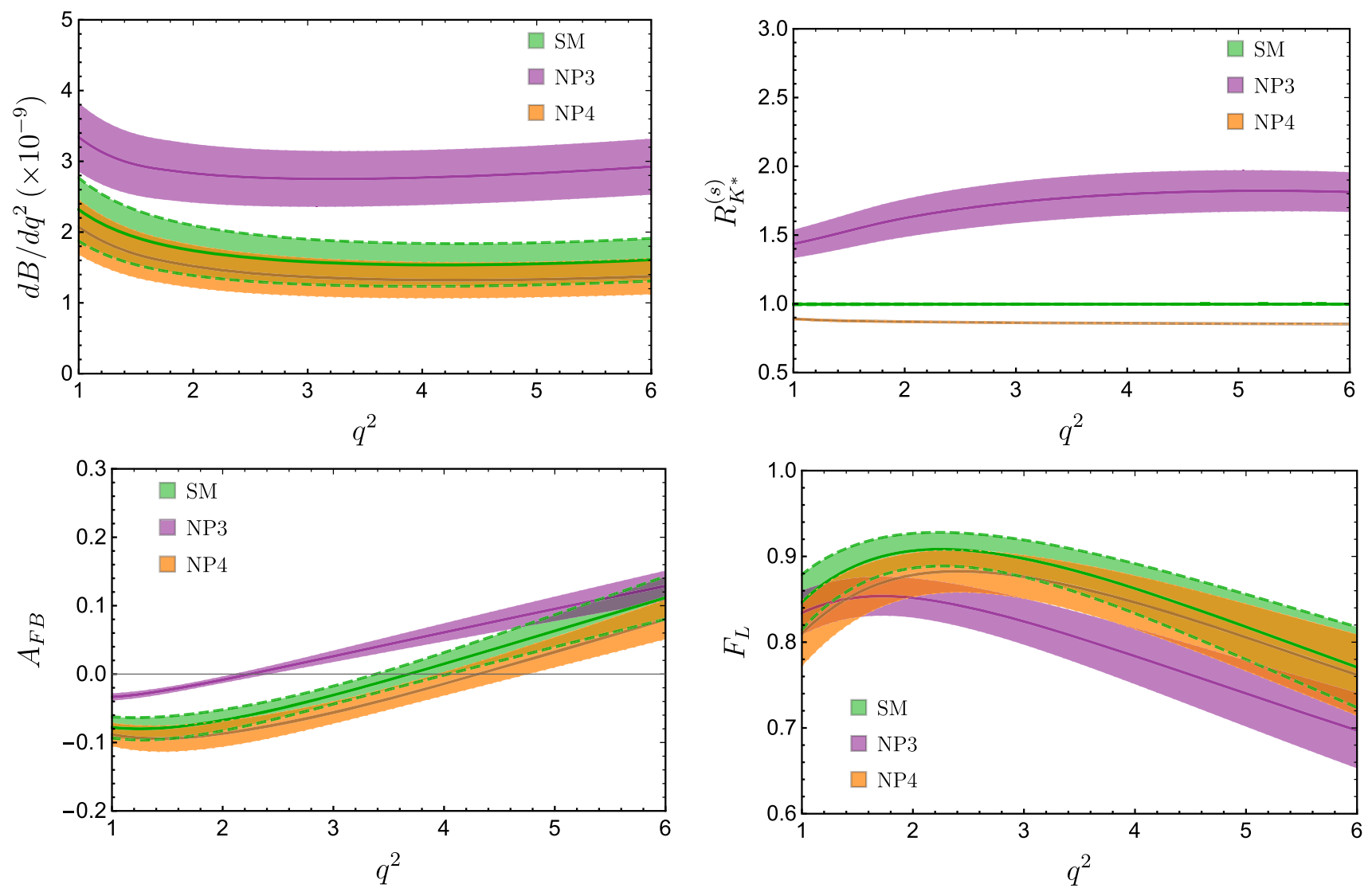

Fig. 6 The predictions for $B_{s} \rightarrow \bar{K}^{*} \mu^{+} \mu^{-}$decay, with complex $Z^{\prime}$ couplings, for SM and the benchmark scenarios NP3 and NP4 as given in Table 1

\subsection{Complex couplings}

We would now like to see how the predictions for the above observables in the $B_{S} \rightarrow \bar{K}^{*} \mu^{+} \mu^{-}$decay would change if the couplings $g_{L}^{b d}$ and $g_{L}^{b s}$ are allowed to be complex. Note that since the leptonic current in Eq. (3) is self-conjugate, $g_{L}^{\mu \mu}$ and $g_{R}^{\mu \mu}$ must be real. We also study the impact of these complex couplings on the direct $C P$ asymmetry in this decay.

Figure 5 shows the $1 \sigma$-favored regions of the couplings $\operatorname{Re}\left[g_{L}^{b d}\right], g_{L}^{\mu \mu}$, and $g_{R}^{\mu \mu}$. The minimum $\chi^{2}$ in the presence of the complex NP couplings is $\chi_{\mathrm{NP}}^{2} \approx 178$, so that $\Delta \chi^{2} \approx 43$, thereby providing a slightly better fit as compared to the case of real couplings $\left(\Delta \chi^{2}=41\right)$. The corresponding best fit values are $\operatorname{Re}\left[g_{L}^{b d}\right]= \pm 2.7 \times 10^{-3}, \operatorname{Im}\left[g_{L}^{b d}\right]=\mp 3.8 \times 10^{-3}$, $g_{L}^{\mu \mu}=\mp 1$ and $g_{R}^{\mu \mu}=\mp 0.255$. As $\chi_{\mathrm{NP}}^{2}$ for complex couplings is lower compared to that for real couplings, the $1 \sigma$ favored parameter space shifts further away from the SM point. A larger parameter space is allowed for the muon couplings compared to the real case, $\left|g_{L}^{\mu \mu}\right| \leq 2.5$ and $\left|g_{R}^{\mu \mu}\right| \leq$ 1.4. The $1 \sigma$ favored region encompasses $g_{R}^{\mu \mu}=0$, whereas a rather large region around $g_{L}^{\mu \mu}=0$ (i.e. $\left|g_{L}^{\mu \mu}\right| \leq 0.4$ ) is disfavoured within $1 \sigma$. The allowed range of $\operatorname{Im}\left[g_{L}^{b d}\right]$ is qualitatively similar to that of $\operatorname{Re}\left[g_{L}^{b d}\right]$. Note that the com- plex nature of $g_{L}^{b d}$ is constrained only from $B_{d}-\bar{B}_{d}$ mixing measurements, since no $C P$-violating measurements are currently available in the $b \rightarrow d \mu \mu$ sector.

\subsubsection{Predictions for $d B / d q^{2}, R_{K^{*}}^{(s)}\left(q^{2}\right), A_{F B}\left(q^{2}\right)$ and $F_{L}\left(q^{2}\right)$}

The predictions for differential branching ratio and $R_{K^{*}}^{(s)}\left(q^{2}\right)$ for the $Z^{\prime}$ model with complex couplings are shown in the top panel of Fig. 6, for SM as well as the benchmark scenarios NP3 and NP4 in Table 1. These scenarios are the $1 \sigma$-favored ones with a maximum value of $\operatorname{Im}\left[C_{9}^{b d, \mathrm{NP}}\right]$ and a minimum value of $\operatorname{Re}\left[C_{9}^{b d, \mathrm{NP}}\right]$, respectively, and are observed to provide close to maximal allowed deviation from the SM predictions. A significant enhancement in the branching ratio is possible in NP3, which could be useful in identifying deviations from the SM. A large enhancement is also possible in the LFUV ratio $R_{K^{*}}^{(s)}\left(q^{2}\right)$ in the NP3 scenario, with the maximum value of $R_{K^{*}}^{(s)}=1.8$ at $q^{2}=6 \mathrm{GeV}^{2}$. While the scenario NP4 cannot be distinguished from the SM using only the branching ratio, the value of $R_{K^{*}}^{(s)}\left(q^{2}\right)$ in this scenario can be as low as 0.85 . Therefore, $R_{K^{*}}^{(s)}\left(q^{2}\right)$ would be useful to identify deviations from the SM. 

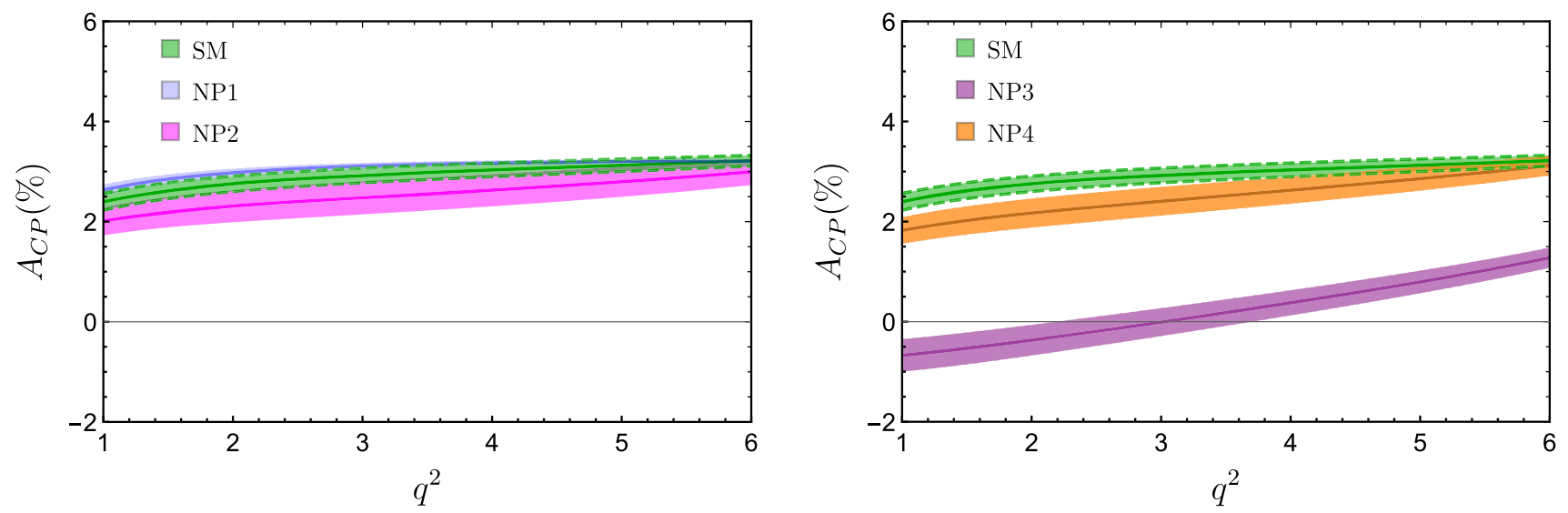

Fig. 7 Predictions for the direct $C P$ asymmetry $A_{C P}\left(q^{2}\right)$ in $B_{S} \rightarrow \bar{K}^{*} \mu^{+} \mu^{-}$decay for benchmark scenarios with real couplings (left) and complex couplings (right)
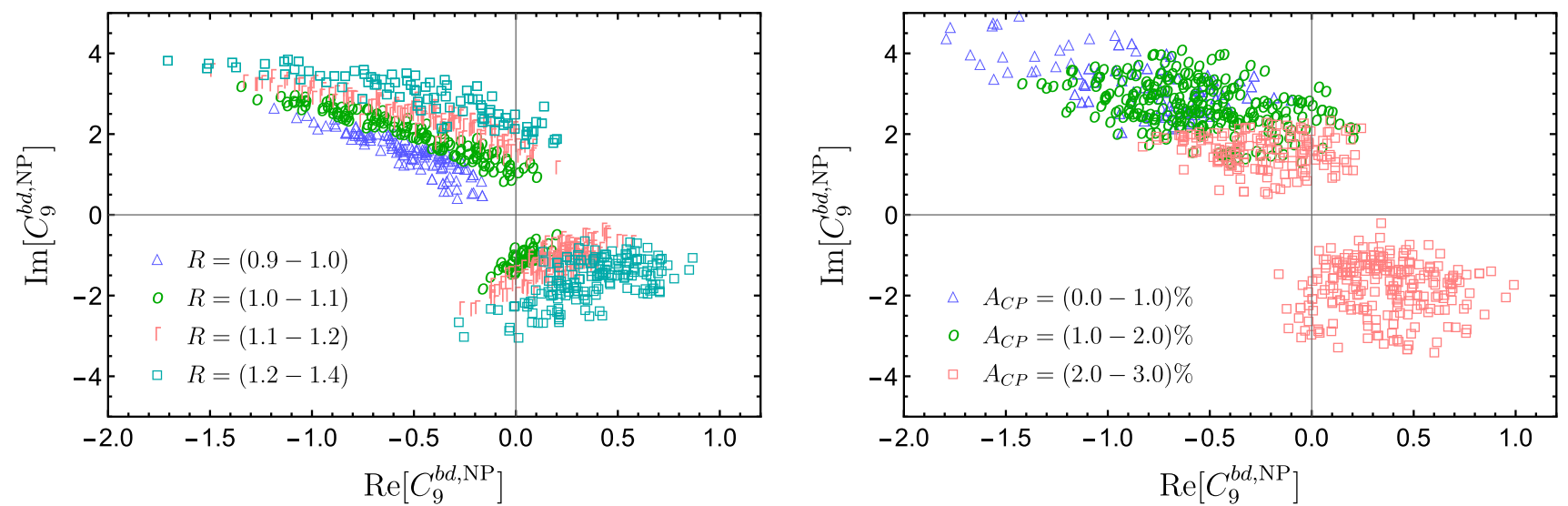

Fig. 8 The integrated values of the LFUV ratio $R_{K^{*}}^{(s)}$ (left panel) and the direct $C P$ asymmetry $A_{C P}$ (right panel) in the $1 \sigma$-favored parameter space of $\left(\operatorname{Re}\left[C_{9}^{b d, \mathrm{NP}}\right], \operatorname{Im}\left[C_{9}^{b d, \mathrm{NP}}\right]\right)$ for the $Z^{\prime}$ model with complex couplings

A marginal enhancement in $A_{F B}\left(q^{2}\right)$ is possible for the scenario NP3, which would also display zero-crossing at much lower $q^{2}$ values $\left(q^{2} \approx 2.5 \mathrm{GeV}^{2}\right)$ compared to that in the $\mathrm{SM}\left(q^{2} \approx 3.5 \mathrm{GeV}^{2}\right)$. A marginal suppression in $F_{L}\left(q^{2}\right)$ is also possible in NP3. The scenario NP4, on the other hand, does not show significant deviations from the SM for these two observables.

\subsubsection{Direct $C P$ asymmetry $A_{C P}\left(q^{2}\right)$}

The direct $C P$ asymmetry in the $b \rightarrow d \mu^{+} \mu^{-}$sector is expected to be about an order of magnitude larger than $b \rightarrow$ $s \mu^{+} \mu^{-}$. As direct $C P$ violation in $b \rightarrow s \mu^{+} \mu^{-}$sector is expected to be $\sim 0.1 \%$, its experimental observation would be possible only if some new physics provides an order of magnitude enhancement to bring it up to the level of a few percent. In $b \rightarrow d \mu^{+} \mu^{-}$decays, the $A_{C P}$ in SM itself is at the level of a few per cent, and can be within experimental reach.
Figure 7 shows $A_{C P}\left(q^{2}\right)$ in the low- $q^{2}$ region for the decay $B_{s} \rightarrow \bar{K}^{*} \mu^{+} \mu^{-}$, considering the benchmark scenarios NP1 and NP2 (real couplings), as well as NP3 and NP4 (complex couplings). It can be seen from the left panel of the figure that for real couplings, $A_{C P}\left(q^{2}\right)$ is either marginally below the SM prediction or almost consistent with it.

For complex couplings, however the suppression in $A_{C P}\left(q^{2}\right)$ can be quite large. It can even lead to $A_{C P}\left(q^{2}\right)$ falling below a per cent level, hence making its measurement extremely difficult. In some scenarios (e.g. NP3), it is even possible for $A_{C P}\left(q^{2}\right)$ to be negative for very low $q^{2}$ values. After scanning over the $1 \sigma$-favored parameter space, we find no significant enhancement in $A_{C P}\left(q^{2}\right)$. So an NP signal can be established if the measurements put an upper bound which is firmly below the SM prediction of $A_{C P}\left(q^{2}\right)$.

\subsubsection{Integrated $R_{K^{*}}^{(s)}$ and $A_{C P}$}

As observed in the case of real couplings, the integrated branching ratio does not help much in narrowing down the 
range of effective NP Wilson coefficients. Hence, in this section, we focus on the integrated values of $R_{K^{*}}^{(s)}$ and $A_{C P}$ over $q^{2}=(1-6) \mathrm{GeV}^{2}$ bin. Figure 8 depicts these results in the $\left(\operatorname{Re}\left[\mathrm{C}_{9}^{\mathrm{bd}, \mathrm{NP}}\right], \operatorname{Im}\left[\mathrm{C}_{9}^{\mathrm{bd}, \mathrm{NP}}\right]\right)$ plane, with different colors and symbols indicating the values of integrated $R_{K^{*}}^{(s)}$ (left panel) and $A_{C P}$ (right panel). At each $1 \sigma$-favored complex value of $\left(C_{9}^{b d, \mathrm{NP}}, C_{10}^{b d, \mathrm{NP}}\right)$, we vary the values of form factor parameters within their $1 \sigma$ range [63] with a Gaussian distribution of uncertainties.

As in the case of real NP couplings, integrated $R_{K^{*}}^{(s)}$ below the SM prediction of unity could indicate a negative value of $\operatorname{Re}\left[C_{9}^{b d, \mathrm{NP}}\right]$. An enhancement in integrated $R_{K^{*}}^{(s)}$ upto $(1.2$ - 1.6) is possible for large positive or negative values of $\operatorname{Im}\left[C_{9}^{b d, \mathrm{NP}}\right]$. These features may be understood from the observation that in the case of complex couplings, $R_{K^{*}}^{(s)}$ has contributions both from $\operatorname{Re}\left[C_{9}^{b d, \mathrm{NP}}\right]$ and $\left|C_{9}^{b d, \mathrm{NP}}\right|^{2}$.

The right panel of Fig. 8 shows that a large positive value of $\operatorname{Im}\left[C_{9}^{b d, \mathrm{NP}}\right]$ can decrease the integrated $A_{C P}$ to less than a per cent. The negative values of $\operatorname{Im}\left[C_{9}^{b d, \mathrm{NP}}\right]$ do not seem to affect $A_{C P}$ much, keeping it close to the SM prediction of $2.5 \%$. Therefore, a simultaneous measurement of integrated $R_{K^{*}}^{(s)}$ and $A_{C P}$, with a precision of 0.1 and $1 \%$, respectively, may help identify the sign of $\operatorname{Im}\left[C_{9}^{b d, \mathrm{NP}}\right]$. We find that the measurements of integrated $R_{K^{*}}^{(s)}$ and $A_{C P}$ values are not very useful in identifying the allowed ranges of $\operatorname{Re}\left[C_{10}^{b d, \mathrm{NP}}\right]$ and $\operatorname{Im}\left[C_{10}^{b d, \mathrm{NP}}\right]$.

\section{Summary and conclusions}

In non-universal $Z^{\prime}$ models, instrumental in accounting for the flavor anomalies, the observables in $b \rightarrow s \ell \ell$ and $b \rightarrow d \ell \ell$ processes would be correlated. In this paper, we study the constraints on the couplings of a non-universal $Z^{\prime}$ model from the measurements in $b \rightarrow q \mu \mu(q=s, d)$ decays, $B_{q}-\bar{B}_{q}$ mixing, and neutrino trident production. These couplings give rise to new additional contributions to the Wilson coefficients $C_{9}^{b q}$ and $C_{10}^{b q}$. Using the above constraints, we perform a global fit to determine $1 \sigma$-favored regions in the parameter space of the $Z^{\prime}$ couplings $g_{L}^{b d}, g_{L}^{b s}$, $g_{L}^{\mu \mu}$, and $g_{R}^{\mu \mu}$. We analyze the cases when quark- $Z^{\prime}$ couplings $g_{L}^{b d}$ and $g_{L}^{b s}$ are (i) real, and (ii) complex. We also present our predictions for some important observables in $B_{S} \rightarrow \bar{K}^{*} \mu \mu$ decays - the differential branching ratio $d B / d q^{2}$, the LFUV ratio $R_{K^{*}}^{(s)}$, the angular observables $A_{F B}$ and $F_{L}$, and the $C P$ asymmetry $A_{C P}$ - for some benchmark scenarios.

It is observed from our analyses that the $Z^{\prime}$ model improves the global fit over the SM by $\Delta \chi^{2} \approx 41$ (real couplings) and $\Delta \chi^{2} \approx 43$ (complex couplings). The favored regions in the parameter space lie along $g_{L}^{\mu \mu} \approx g_{R}^{\mu \mu}$, corresponding to $C_{10}^{b d, \mathrm{NP}} \approx 0$, while the region around $g_{L}^{\mu \mu}=0$ is disfavored. These are mainly dictated by the $R_{K}$ and $R_{K} *$ anomalies in $b \rightarrow s$ sector.

For the observables in $B_{S} \rightarrow \bar{K}^{*} \mu^{+} \mu^{-}$decays, when the couplings are real, we find that the enhancement and suppression in $d B / d q^{2}$ cannot be cleanly identified due to the large uncertainties in the SM prediction. However, the value of $R_{K^{*}}^{(s)}\left(q^{2}\right)$ can substantially deviate from the SM prediction of unity - it can range from 0.8 to 1.3 . The enhancement (suppression) corresponds to positive (negative) values of $C_{9}^{b d, \mathrm{NP}}$. A marginal enhancement and suppression in $A_{F B}\left(q^{2}\right)$ is possible compared to the SM predictions, with the zero-crossing shifting towards lower (higher) $q^{2}$ values for positive (negative) values of $C_{9}^{b d, \mathrm{NP}}$. There is no significant deviation from SM in the predictions of $F_{L}\left(q^{2}\right)$, and the predictions of $A_{C P}\left(q^{2}\right)$ also stay close to the SM expectation for all the favored values of NP Wilson coefficients. Further, we find that a measurement of integrated $R_{K^{*}}^{(s)}$ in the low- $q^{2}$ bin with a precision of $\sim 0.1$ can help narrow down the ranges of $\left(C_{9}^{b d, \mathrm{NP}}, C_{10}^{b d, \mathrm{NP}}\right)$.

In the case of complex couplings, a larger NP parameter space is allowed, leading to larger possible deviations in the $B_{s} \rightarrow \bar{K}^{*} \mu \mu$ observables. In particular, a $\sim 50 \%$ enhancement in $d B / d q^{2}$ is allowed. Moreover, the LFUV ratio $R_{K^{*}}^{(s)}\left(q^{2}\right)$ can be enhanced up to 1.8 in scenarios with large positive and negative $\operatorname{Im}\left[C_{9}^{b d, \mathrm{NP}}\right]$. There can also be a significant enhancement in $A_{F B}\left(q^{2}\right)$ for positive values of $\operatorname{Re}\left[C_{9}^{b d, \mathrm{NP}}\right]$ and large $\operatorname{Im}\left[C_{9}^{b d, \mathrm{NP}}\right]$, with the zerocrossing shifting towards lower $q^{2}$. A significant suppression in $A_{C P}\left(q^{2}\right)$ compared to the $\mathrm{SM}$ prediction of $2.5 \%$ is possible for large positive values of $\operatorname{Im}\left[C_{9}^{b d, \mathrm{NP}}\right]$, which may lead to $A_{C P}\left(q^{2}\right)$ falling below a per cent level. We find that a measurement of integrated $R_{K^{*}}^{(s)}$ and $A_{C P}$, with a precision 0.1 and $1 \%$, respectively, would be needed to narrow down the allowed ranges of $\left(\operatorname{Re}\left[C_{9}^{b d, \mathrm{NP}}\right], \operatorname{Im}\left[C_{9}^{b d, \mathrm{NP}}\right]\right)$.

To summarize, we study NP effects in $B_{s} \rightarrow \bar{K}^{*} \ell \ell$ decays in a generic $Z^{\prime}$ model with real as well as complex couplings. The constraints on the $Z^{\prime}$ couplings are obtained by correlating measurements in the $b \rightarrow s$ and $b \rightarrow d$ sectors, along with neutrino trident production. We find that

- The present data allow a large deviation (enhancement as well as suppression) in $R_{K^{*}}^{(s)}$ from its SM prediction. The deviation is more pronounced for complex NP couplings.

- The $C P$ asymmetry can be significantly suppressed as compared to the SM prediction.

The modes $B_{S} \rightarrow \bar{K}^{*} \ell \ell$ are expected to be measured with a good accuracy in the near future. The observables $R_{K^{*}}^{(s)}$ and $A_{C P}$ in $B_{s} \rightarrow \bar{K}^{*} \mu \mu$ decays can show clean signatures of the presence of NP. Hence their measurements will be crucial in the search for physics beyond the SM. 


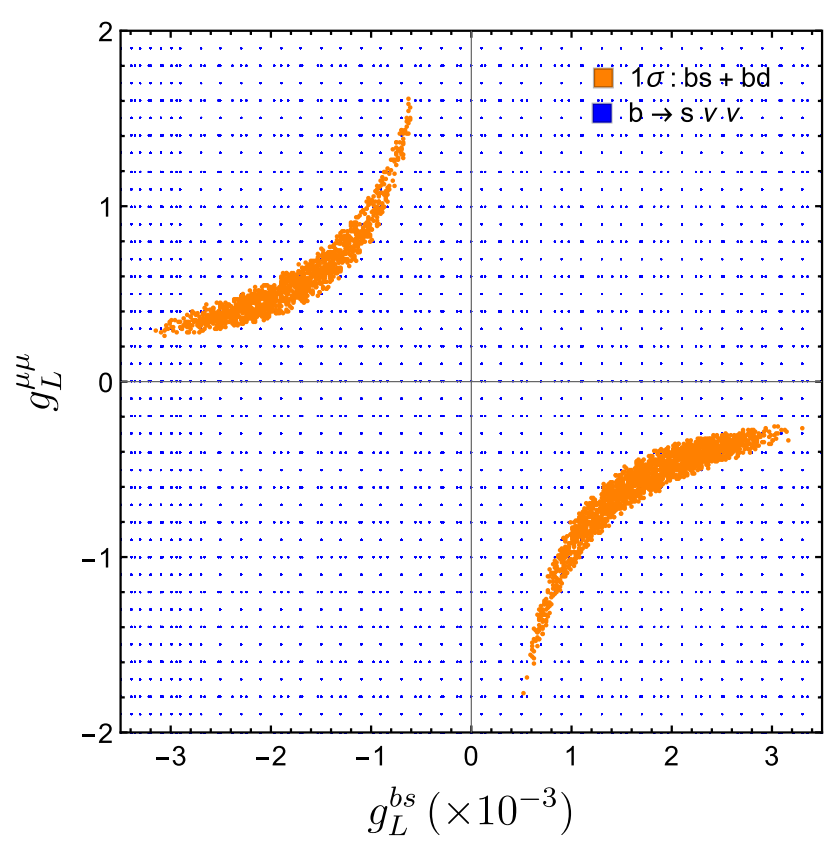

Fig. 9 Comparison of favored NP parameter space using $b \rightarrow s v \bar{v}$ data and combined fit to $b \rightarrow s, b \rightarrow d$ and neutrino trident data

Acknowledgements We would like to thank Suman Kumbhakar for his contribution during the initial stages of this work. The work of DK is supported by the National Science Centre (Poland) under the research Grant no. 2017/26/E/ST2/00470. We would like to thank the organizers of WHEPP 2019, where this work was completed.

Data Availability Statement This manuscript has no associated data or the data will not be deposited. [Authors' comment: This is a theoretical work. The experimental inputs used in our analysis are in the public domain and we have cited the sources of these inputs in the references.]

Open Access This article is licensed under a Creative Commons Attribution 4.0 International License, which permits use, sharing, adaptation, distribution and reproduction in any medium or format, as long as you give appropriate credit to the original author(s) and the source, provide a link to the Creative Commons licence, and indicate if changes were made. The images or other third party material in this article are included in the article's Creative Commons licence, unless indicated otherwise in a credit line to the material. If material is not included in the article's Creative Commons licence and your intended use is not permitted by statutory regulation or exceeds the permitted use, you will need to obtain permission directly from the copyright holder. To view a copy of this licence, visit http://creativecomm ons.org/licenses/by/4.0/.

Funded by SCOAP ${ }^{3}$.

\section{Appendix A: Constraints from $b \rightarrow s v \bar{v}$}

The quark level transition $b \rightarrow s v \bar{v}$ induces exclusive semileptonic decays $B \rightarrow K^{(*)} v v$. The effective Hamiltonian relevant for $b \rightarrow s v \bar{v}$ transition is [82]

$H_{\mathrm{eff}}=-\frac{\sqrt{2} \alpha G_{F}}{\pi} V_{t b} V_{t s}^{*} \sum_{\ell} C_{L}^{\ell}\left(\bar{s} \gamma_{\mu} P_{L} b\right)\left(\bar{v}_{\ell} \gamma^{\mu} P_{L} v_{\ell}\right)$, where $C_{L}^{\ell}=C_{L}^{\mathrm{SM}}+C_{v}^{\ell \ell}(\mathrm{NP})$. The NP contribution $C_{v}^{\mu \mu}(\mathrm{NP})$ in the $Z^{\prime}$ model is given by

$C_{\nu}^{\mu \mu}(\mathrm{NP})=-\frac{\pi}{\sqrt{2} G_{F} \alpha V_{t b} V_{t s}^{*}} \frac{g_{L}^{b s} g_{L}^{\mu \mu}}{M_{Z^{\prime}}^{2}}$.

The SM WC is $C_{L}^{\mathrm{SM}}=-X_{t} / s_{W}^{2}$, where $s_{W} \equiv \sin \theta_{W}$ and $X_{t}=1.469 \pm 0.017$.

From the experimental side, at present, we only have following upper limits [83-87]

$$
\begin{aligned}
\mathcal{B}\left(B^{0} \rightarrow K^{0} \nu \bar{v}\right) & <2.9 \times 10^{-5}, \\
\mathcal{B}\left(B^{0} \rightarrow K^{* 0} \nu \bar{v}\right) & <2.0 \times 10^{-5}, \\
\mathcal{B}\left(B^{+} \rightarrow K^{+} \nu \bar{v}\right) & <1.7 \times 10^{-5}, \\
\mathcal{B}\left(B^{+} \rightarrow K^{*+} \nu \bar{\nu}\right) & <4.8 \times 10^{-5} .
\end{aligned}
$$

Using the above bounds, the allowed NP parameter space from $b \rightarrow s v \bar{v}$ data near our best-fit region is depicted in Fig. 9. It is evident that the bounds coming from the current $B \rightarrow K^{(*)} \nu v$ data are much weaker than those obtained from the combined $b \rightarrow s, b \rightarrow d$ and neutrino-trident fit.

\section{Appendix B: Constraints from $D^{0}-\bar{D}^{0}$ mixing and $D^{0} \rightarrow$ $\mu^{+} \mu^{-}$decay}

The quark doublets in Eq. (2) are taken to be in the downtype quark diagonal basis. Hence owing to quark mixing, the up-type quarks in the quark doublets induce $u_{i} \rightarrow u_{j}$ transitions. Then there can be constraints coming from the up quark sector, in particular $D^{0}-\bar{D}^{0}$ mixing and charm decays. The relevant terms in the effective Hamiltonian for the $c \rightarrow u$ sector are

$$
\begin{aligned}
\mathcal{H}_{\mathrm{eff}}^{Z^{\prime}, c \rightarrow u} \supset & \frac{1}{2 M_{Z^{\prime}}^{2}} J_{\alpha} J^{\alpha}=\frac{g_{L}^{c u}}{2 M_{Z^{\prime}}^{2}}\left(\bar{u} \gamma^{\alpha} P_{L} c\right)\left(\bar{u} \gamma_{\alpha} P_{L} c\right) \\
& +\frac{h_{L}^{c u}}{M_{Z^{\prime}}^{2}}\left(\bar{u} \gamma^{\alpha} P_{L} c\right)\left[\bar{\mu} \gamma_{\alpha}\left(g_{L}^{\mu \mu} P_{L}+g_{R}^{\mu \mu} P_{R}\right) \mu\right],
\end{aligned}
$$

where

$$
\begin{aligned}
g_{L}^{c u}= & \left(g_{L}^{b s} V_{u d} V_{c b}^{*}\right)^{2}+\left(g_{L}^{b d} V_{u s} V_{c b}^{*}\right)^{2} \\
& +2 g_{L}^{b s} g_{L}^{b d} V_{u d} V_{c b}^{*} V_{u s} V_{c b}^{*}, \\
h_{L}^{c u}= & g_{L}^{b s} V_{u d} V_{c b}^{*}+g_{L}^{b d} V_{u s} V_{c b}^{*} .
\end{aligned}
$$

The first term in Eq. (B.4) induces $D^{0}-\bar{D}^{0}$ mixing whereas the second term induces $c \rightarrow u \mu^{+} \mu^{-}$transition. Here we consider constraints from $D-\bar{D}$ mixing and $D^{0} \rightarrow \mu^{+} \mu^{-}$.

In the $\mathrm{SM}, D^{0}-\bar{D}^{0}$ mixing is induced at the loop level by the quarks d, s and b. Due to a strong GIM cancellation, the short-distance contribution is extremely small. In particular, the contribution due to b-quark is highly suppressed, $O\left(\lambda^{8}\right)$. Therefore $D^{0}-\bar{D}^{0}$ mixing is dominated by the d- and s-quarks 

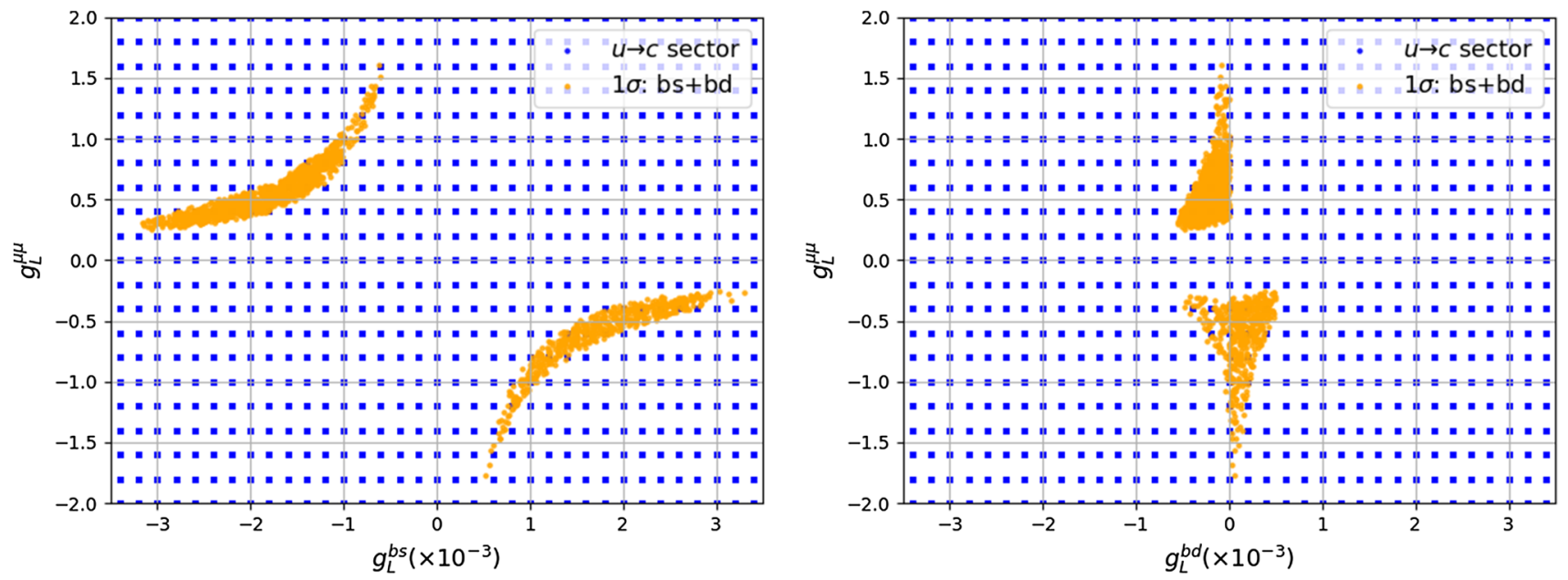

Fig. 10 Comparison of allowed NP parameter space using $D^{0}-\bar{D}^{0}$ mixing \& branching ratio of $D^{0} \rightarrow \mu^{+} \mu^{-}$and combined $b \rightarrow s, b \rightarrow d$ and neutrino trident fit

and hence there can be large long-distance contributions, for which there are no reliable estimates at present $[88,89]$. In our analysis, we consider the $D^{0}-\bar{D}^{0}$ mixing parameter $\Delta M_{D}$ which is measured to be $0.0095_{-0.0044}^{+0.0041} \mathrm{ps}^{-1}$ [67]. In $Z^{\prime}$ model, $D^{0}-\bar{D}^{0}$ mixing is induced at the tree level and hence would provide a much larger contribution in comparison to the short-distance SM contribution. Further as longdistance contributions are unknown, we saturate the $\Delta M_{D}$ experimental value with new physics contribution which is given by

$\Delta M_{D}=\frac{f_{D}^{2} m_{D} B_{D} r\left(m_{c}, M_{Z^{\prime}}\right)}{3 M_{Z^{\prime}}^{2}}\left(g_{L}^{c u}\right)^{2}$,

where $f_{D}=212.0 \pm 0.7 \mathrm{MeV}[66], B_{D}=0.757 \pm 0.027 \pm$ 0.004 [90] and $r\left(m_{c}, M_{Z^{\prime}}\right)=0.72$ for $M_{Z^{\prime}}=1 \mathrm{TeV}$ [91].

The decay $D^{0} \rightarrow \mu^{+} \mu^{-}$is induced by the quark level transition $c \rightarrow u \mu^{+} \mu^{-}$. In $Z^{\prime}$ model, the branching ratio is given by

$$
\begin{aligned}
B\left(D^{0} \rightarrow \mu^{+} \mu^{-}\right)= & \frac{\tau_{D} f_{D}^{2} m_{\mu}^{2} m_{D}}{32 \pi M_{Z^{\prime}}^{4}} \sqrt{1-\frac{4 m_{\mu}^{2}}{m_{D}^{2}}} \\
& \times\left(h_{L}^{c u}\right)^{2}\left(g_{L}^{\mu \mu}-g_{R}^{\mu \mu}\right)^{2} .
\end{aligned}
$$

Within the SM, $D^{0} \rightarrow \mu^{+} \mu^{-}$is dominated by the intermediate $\gamma^{*} \gamma^{*}$ state which scales its branching ratio as $2.7 \times 10^{-5}$ times the branching ratio for $D^{0} \rightarrow \gamma \gamma$ [92]. Using the upper bound on $D^{0} \rightarrow \gamma \gamma<2.2 \times 10^{-6}$ at $90 \%$ C.L. [93], the SM branching ratio is estimated to be $\lesssim 10^{-10}$. From the experimental side, we only have an upper bound which is $<6.2 \times 10^{-9}$ at $90 \%$ C.L. [94].

Figure 10 shows the region allowed by the branching ratio of $D^{0} \rightarrow \mu^{+} \mu^{-}$and $\Delta M_{D}$ as well as from the combined fit in the region around our best-fit point. It is obvious that the constraints coming from the charm sector are significantly weaker.

\section{References}

1. G. Hiller, F. Kruger, More model-independent analysis of $b \rightarrow s$ processes. Phys. Rev. D 69, 074020 (2004). arXiv:hep-ph/0310219

2. M. Bordone, G. Isidori and A. Pattori, "On the standard model predictions for $R_{K}$ and $R_{K}$ *”, Eur. Phys. J. C 76, no. 8, 440 (2016). arXiv:1605.07633 [hep-ph]

3. R. Aaij et al., [LHCb Collaboration], "Test of lepton universality with $B^{0} \rightarrow K^{* 0} \ell^{+} \ell^{-}$decays". JHEP 1708, 055 (2017). arXiv:1705.05802 [hep-ex]

4. R. Aaij et al. [LHCb Collaboration], "Search for lepton-universality violation in $B^{+} \rightarrow K^{+} \ell^{+} \ell^{-}$decays", Phys. Rev. Lett. 122, no. 19, 191801 (2019). arXiv:1903.09252 [hep-ex]

5. D. Bhatia, S. Chakraborty, A. Dighe, Neutrino mixing and $R_{K}$ anomaly in $\mathrm{U}(1)_{X}$ models: a bottom-up approach. JHEP 1703, 117 (2017). arXiv:1701.05825 [hep-ph]

6. B. Capdevila, A. Crivellin, S. Descotes-Genon, J. Matias, J. Virto, Patterns of new physics in $b \rightarrow s \ell^{+} \ell^{-}$transitions in the light of recent data. JHEP 1801, 093 (2018). arXiv:1704.05340 [hep-ph]

7. J. Kumar and D. London, "New physics in $b \rightarrow s e^{+} e^{-}$?". arXiv:1901.04516 [hep-ph]

8. R. Aaij et al., [LHCb Collaboration], "Angular analysis and differential branching fraction of the decay $B_{s}^{0} \rightarrow \phi \mu^{+} \mu^{-}$". JHEP 1509, 179 (2015). arXiv:1506.08777 [hep-ex]

9. R. Aaij et al., [LHCb Collaboration], "Measurement of form-factorindependent observables in the decay $B^{0} \rightarrow K^{* 0} \mu^{+} \mu^{-}$". Phys. Rev. Lett. 111, 191801 (2013). arXiv:1308.1707 [hep-ex]

10. R. Aaij et al., [LHCb Collaboration], "Angular analysis of the $B^{0} \rightarrow K^{* 0} \mu^{+} \mu^{-}$decay using $3 \mathrm{fb}^{-1}$ of integrated luminosity". JHEP 1602, 104 (2016). arXiv:1512.04442 [hep-ex]

11. S. Descotes-Genon, T. Hurth, J. Matias, J. Virto, Optimizing the basis of $B \rightarrow K^{*} l l$ observables in the full kinematic range. JHEP 1305, 137 (2013). arXiv:1303.5794 [hep-ph]

12. M. Algueró, B. Capdevila, A. Crivellin, S. Descotes-Genon, P. Masjuan, J. Matias and J. Virto, "Emerging patterns of new physics with and without lepton flavour universal contributions". arXiv:1903.09578 [hep-ph] 
13. A.K. Alok, A. Dighe, S. Gangal, D. Kumar, Continuing search for new physics in $b \rightarrow s \mu \mu$ decays: two operators at a time. JHEP 1906, 089 (2019). arXiv:1903.09617 [hep-ph]

14. M. Ciuchini, A. M. Coutinho, M. Fedele, E. Franco, A. Paul, L. Silvestrini and M. Valli, "New physics in $b \rightarrow s \ell^{+} \ell^{-}$confronts new data on Lepton Universality. arXiv:1903.09632 [hep-ph]

15. G. D'Amico, M. Nardecchia, P. Panci, F. Sannino, A. Strumia, R. Torre, A. Urbano, Flavour anomalies after the $R_{K} *$ measurement. JHEP 1709, 010 (2017). arXiv:1704.05438 [hep-ph]

16. A. Datta, J. Kumar and D. London, "The $B$ anomalies and new physics in $b \rightarrow s e^{+} e^{-"}$. arXiv:1903.10086 [hep-ph]

17. J. Aebischer, W. Altmannshofer, D. Guadagnoli, M. Reboud, P. Stangl and D. M. Straub, "B-decay discrepancies after Moriond 2019”. arXiv:1903.10434 [hep-ph]

18. K. Kowalska, D. Kumar and E. M. Sessolo, "Implications for new physics in $b \rightarrow s \mu \mu$ transitions after recent measurements by Belle and LHCb", Eur. Phys. J. C 79, no. 10, 840 (2019). arXiv:1903.10932 [hep-ph]

19. A. Arbey, T. Hurth, F. Mahmoudi, D. M. Santos and S. Neshatpour, "Update on the $b \rightarrow s$ anomalies", Phys. Rev. D 100, no. 1, 015045 (2019). arXiv:1904.08399 [hep-ph]

20. S. Bhattacharya, A. Biswas, S. Nandi and S. K. Patra, "Exhaustive

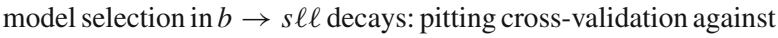
$\mathrm{AIC}_{c}$ ". arXiv:1908.04835 [hep-ph]

21. R. Coy, M. Frigerio, F. Mescia and O. Sumensari, "New physics in $b \rightarrow s \ell \ell$ transitions at one loop", Eur. Phys. J. C 80, no. 1, 52 (2020). arXiv:1909.08567 [hep-ph]

22. A. Crivellin, G. D'Ambrosio and J. Heeck, "Addressing the LHC flavor anomalies with horizontal gauge symmetries", Phys. Rev. D 91, no. 7, 075006 (2015). arXiv:1503.03477 [hep-ph]

23. S.M. Boucenna, A. Celis, J. Fuentes-Martin, A. Vicente, J. Virto, Non-Abelian gauge extensions for B-decay anomalies. Phys. Lett. B 760, 214 (2016). arXiv:1604.03088 [hep-ph]

24. S.M. Boucenna, A. Celis, J. Fuentes-Martin, A. Vicente, J. Virto, Phenomenology of an $S U(2) \times S U(2) \times U(1)$ model with lepton-flavour non-universality. JHEP 1612, 059 (2016). arXiv:1608.01349 [hep-ph]

25. W. Altmannshofer, S. Gori, M. Pospelov, I. Yavin, Quark flavor transitions in $L_{\mu}-L_{\tau}$ models. Phys. Rev. D 89, 095033 (2014). arXiv:1403.1269 [hep-ph]

26. L. Darmé, K. Kowalska, L. Roszkowski, E.M. Sessolo, Flavor anomalies and dark matter in SUSY with an extra U(1). JHEP 1810, 052 (2018). arXiv:1806.06036 [hep-ph]

27. B. Gripaios, M. Nardecchia, S.A. Renner, Composite leptoquarks and anomalies in $B$-meson decays. JHEP 1505, 006 (2015). arXiv:1412.1791 [hep-ph]

28. S. Fajfer, N. Košnik, Vector leptoquark resolution of $R_{K}$ and $R_{D^{(*)}}$ puzzles. Phys. Lett. B 755, 270 (2016). arXiv: 1511.06024 [hep-ph]

29. I. de Medeiros Varzielas and G. Hiller, "Clues for flavor from rare lepton and quark decays", JHEP 1506, 072 (2015). arXiv:1503.01084 [hep-ph]

30. R. Alonso, B. Grinstein and J. Martin Camalich, "Lepton universality violation and lepton flavor conservation in $B$-meson decays", JHEP 1510, 184 (2015). arXiv:1505.05164 [hep-ph]

31. L. Calibbi, A. Crivellin, T. Ota, Effective field theory approach to $b \rightarrow \operatorname{sll}^{\left({ }^{\prime}\right)}, B \rightarrow K^{(*)} v \bar{v}$ and $B \rightarrow D^{(*)} \tau v$ with Third Generation Couplings. Phys. Rev. Lett. 115, 181801 (2015). arXiv:1506.02661 [hep-ph]

32. R. Barbieri, G. Isidori, A. Pattori and F. Senia, "Anomalies in $B$ decays and $U(2)$ flavour symmetry", Eur. Phys. J. C 76, no. 2, 67 (2016). arXiv:1512.01560 [hep-ph]

33. A. Datta, J. Liao, D. Marfatia, A light $Z^{\prime}$ for the $R_{K}$ puzzle and nonstandard neutrino interactions. Phys. Lett. B 768, 265 (2017). arXiv:1702.01099 [hep-ph]
34. A.J. Buras, J. Girrbach, Left-handed $Z^{\prime}$ and $Z$ FCNC quark couplings facing new $b \rightarrow s \mu^{+} \mu^{-}$data. JHEP 1312, 009 (2013). arXiv:1309.2466 [hep-ph]

35. D. Aristizabal Sierra, F. Staub and A. Vicente, "Shedding light on the $b \rightarrow s$ anomalies with a dark sector" Phys. Rev. D 92, no. 1, 015001 (2015). arXiv:1503.06077 [hep-ph]

36. B. Allanach, F. S. Queiroz, A. Strumia and S. Sun, " $Z$ ' models for the LHCb and $g-2$ muon anomalies", Phys. Rev. D 93, no. 5, 055045 (2016). arXiv:1511.07447 [hep-ph]

37. L. Calibbi, A. Crivellin, F. Kirk, C. A. Manzari and L. Vernazza, " $Z$ ' models with less-minimal flavour violation". arXiv:1910.00014 [hep-ph]

38. Q. Chang, X.Q. Li, Y.D. Yang, $B \rightarrow K^{*} l^{+} l^{-}, K l^{+} l^{-}$decays in a family non-universal $Z^{\prime}$ model. JHEP 1004, 052 (2010). arXiv: 1002.2758 [hep-ph]

39. Q. Chang, X.Q. Li, Y.D. Yang, A comprehensive analysis of hadronic $\mathrm{b} \rightarrow \mathrm{s}$ transitions in a family non-universal Z-prime model. J. Phys. G 41, 105002 (2014). arXiv:1312.1302 [hep-ph]

40. R. Aaij et al., [LHCb Collaboration], "First observation of the decay $B^{+} \rightarrow \pi^{+} \mu^{+} \mu^{-}$.. JHEP 1212, 125 (2012). arXiv: 1210.2645 [hep-ex]

41. R. Aaij et al., [LHCb Collaboration], "First measurement of the differential branching fraction and $C P$ asymmetry of the $B^{ \pm} \rightarrow$ $\pi^{ \pm} \mu^{+} \mu^{-}$decay". JHEP 1510, 034 (2015). arXiv:1509.00414 [hep-ex]

42. R. Aaij et al., [LHCb Collaboration], "Evidence for the decay $B_{S}^{0} \rightarrow \bar{K}^{* 0} \mu^{+} \mu^{-”}$. JHEP 1807, 020 (2018). arXiv:1804.07167 [hep-ex]

43. J.-T. Wei et al., [Belle Collaboration], "Search for $B \rightarrow$ $\pi l^{+} l^{-}$Decays at Belle". Phys. Rev. D 78, 011101 (2008). arXiv:0804.3656 [hep-ex]

44. J. P. Lees et al. [BaBar Collaboration], "Search for the rare decays $B \rightarrow \pi l^{+} l^{-}$and $B^{0} \rightarrow \eta l^{+} l^{-}$, , Phys. Rev. D 88, no. 3, 032012 (2013). arXiv:1303.6010 [hep-ex]

45. A. Cerri et al., "Opportunities in flavour physics at the HL-LHC and HE-LHC". arXiv:1812.07638 [hep-ph]

46. V. Barger, L. Everett, J. Jiang, P. Langacker, T. Liu, C. Wagner, Family non-universal U(1)-prime gauge symmetries and $b \rightarrow s$ transitions. Phys. Rev. D 80, 055008 (2009). arXiv:0902. 4507 [hep$\mathrm{ph}$ ]

47. V. Barger, L.L. Everett, J. Jiang, P. Langacker, T. Liu, C.E.M. Wagner, $b \rightarrow s$ Transitions in family-dependent $\mathrm{U}(1)$-prime models. JHEP 0912, 048 (2009). arXiv:0906.3745 [hep-ph]

48. G. Buchalla, A.J. Buras, M.E. Lautenbacher, Weak decays beyond leading logarithms. Rev. Mod. Phys. 68, 1125 (1996). arXiv:hep-ph/9512380

49. A. K. Alok, B. Bhattacharya, D. Kumar, J. Kumar, D. London and S. U. Sankar, "New physics in $b \rightarrow s \mu^{+} \mu^{-}$: Distinguishing models through CP-violating effects", Phys. Rev. D 96 (2017) no.1, 015034 arXiv:1703.09247 [hep-ph]

50. R. Aaij et al., [LHCb Collaboration], "Measurement of the $B_{s}^{0} \rightarrow$ $\mu^{+} \mu^{-}$branching fraction and search for $B^{0} \rightarrow \mu^{+} \mu^{-}$decays at the LHCb experiment". Phys. Rev. Lett. 111, 101805 (2013). arXiv:1307.5024 [hep-ex]

51. V. Khachatryan et al., [CMS and LHCb Collaborations], "Observation of the rare $B_{s}^{0} \rightarrow \mu^{+} \mu^{-}$decay from the combined analysis of CMS and LHCb data". Nature 522, 68 (2015). arXiv:1411.4413 [hep-ex]

52. M. Aaboud et al., [ATLAS Collaboration], "Study of the rare decays of $B_{s}^{0}$ and $B^{0}$ mesons into muon pairs using data collected during 2015 and 2016 with the ATLAS detector". JHEP 1904, 098 (2019). arXiv:1812.03017 [hep-ex]

53. A. Abdesselam et al. [Belle Collaboration], "Test of lepton flavor universality in $B \rightarrow K^{*} \ell^{+} \ell^{-}$decays at Belle". arXiv:1904.02440 [hep-ex] 
54. R. Aaij et al., [LHCb Collaboration], "Measurements of the Swave fraction in $B^{0} \rightarrow K^{+} \pi^{-} \mu^{+} \mu^{-}$decays and the $B^{0} \rightarrow$ $K^{*}(892)^{0} \mu^{+} \mu^{-}$differential branching fraction". JHEP 1611, 047 (2016). arXiv:1606.04731 [hep-ex]

55. CDF Collaboration, "Updated branching ratio measurements of exclusive $b \rightarrow s \mu^{+} \mu^{-}$decays and angular analysis in $B \rightarrow$ $K^{(*)} \mu^{+} \mu^{-}$Decays", CDF public note 10894

56. S. Chatrchyan et al., [CMS Collaboration], "Angular analysis and branching fraction measurement of the decay $B^{0} \rightarrow K^{* 0} \mu^{+} \mu^{- \text {" }}$. Phys. Lett. B 727, 77 (2013). arXiv:1308.3409 [hep-ex]

57. V. Khachatryan et al., [CMS Collaboration], "Angular analysis of the decay $B^{0} \rightarrow K^{* 0} \mu^{+} \mu^{-}$from pp collisions at $\sqrt{s}=8 \mathrm{TeV}$ ". Phys. Lett. B 753, 424 (2016). arXiv:1507.08126 [hep-ex]

58. R. Aaij et al., [LHCb Collaboration], "Differential branching fractions and isospin asymmetries of $B \rightarrow K^{(*)} \mu^{+} \mu^{-}$decays". JHEP 1406, 133 (2014). arXiv:1403.8044 [hep-ex]

59. J.P. Lees et al., [BaBar Collaboration], "Measurement of the $B \rightarrow$ $X_{S} l^{+} l^{-}$branching fraction and search for direct CP violation from a sum of exclusive final states”. Phys. Rev. Lett. 112, 211802 (2014). arXiv:1312.5364 [hep-ex]

60. M. Aaboud et al., [ATLAS Collaboration], "Angular analysis of $B_{d}^{0} \rightarrow K^{*} \mu^{+} \mu^{-}$decays in $p p$ collisions at $\sqrt{s}=8 \mathrm{TeV}$ with the ATLAS detector ". JHEP 1810, 047 (2018). arXiv: 1805.04000 [hep-ex]

61. CMS Collaboration [CMS Collaboration], "Measurement of the $P_{1}$ and $P_{5}^{\prime}$ angular parameters of the decay $\mathrm{B}^{0} \rightarrow \mathrm{K}^{* 0} \mu^{+} \mu^{-}$in proton-proton collisions at $\sqrt{s}=8 \mathrm{TeV}$ ", CMS-PAS-BPH-15-008

62. N. Gubernari, A. Kokulu, D. van Dyk, $B \rightarrow P$ and $B \rightarrow V$ form factors from $B$-meson light-cone sum rules beyond leading twist. JHEP 1901, 150 (2019). arXiv:1811.00983 [hep-ph]

63. A. Bharucha, D.M. Straub, R. Zwicky, $B \rightarrow V \ell^{+} \ell^{-}$in the standard model from light-cone sum rules. JHEP 1608, 098 (2016). arXiv:1503.05534 [hep-ph]

64. D. M. Straub, "flavio: a Python package for flavour and precision phenomenology in the standard model and beyond". arXiv:1810.08132 [hep-ph]

65. A. Khodjamirian, T. Mannel, A.A. Pivovarov, Y.-M. Wang, Charmloop effect in $B \rightarrow K^{(*)} \ell^{+} \ell^{-}$and $B \rightarrow K^{*} \gamma$. JHEP 1009, 089 (2010). arXiv:1006.4945 [hep-ph]

66. S. Aoki et al. [Flavour Lattice Averaging Group], "FLAG Review 2019”. arXiv:1902.08191 [hep-lat]

67. M. Tanabashi et al. [Particle Data Group], Review of particle physics, Phys. Rev. D 98, no. 3, 030001 (2018)

68. Y. S. Amhis et al. [Heavy Flavor Averaging Group], "Averages of $b$-hadron, $c$-hadron, and $\tau$-lepton properties as of 2018". arXiv:1909.12524 [hep-ex]

69. D. King, A. Lenz, T. Rauh, $\mathrm{B}_{s}$ mixing observables and $\left|V_{t d} / V_{t s}\right|$ from sum rules. JHEP 1905, 034 (2019). arXiv:1904.00940 [hep$\mathrm{ph}]$

70. J. Charles et al. [CKMfitter Group], "CP violation and the CKM matrix: assessing the impact of the asymmetric $B$ factories", Eur. Phys. J. C 41, no. 1, 1 (2005) arXiv:hep-ph/0406184

71. S.R. Mishra et al., [CCFR Collaboration], "Neutrino tridents and W Z interference". Phys. Rev. Lett. 66, 3117 (1991)

72. W. Altmannshofer, S. Gori, J. Martín-Albo, A. Sousa and M. Wallbank, "Neutrino tridents at DUNE". arXiv:1902.06765 [hep-ph]

73. J.J. Wang, R.M. Wang, Y.G. Xu, Y.D. Yang, The rare decays $B_{u}^{+} \rightarrow \pi^{+} l^{+} l^{-}, \rho^{+} l^{+} l^{-}$and $B_{d}^{0} \rightarrow l^{+} l^{-}$in the R-parity violating supersymmetry. Phys. Rev. D 77, 014017 (2008). arXiv:0711.0321 [hep-ph]

74. P. Ball, R. Zwicky, New results on $B \rightarrow \pi, K, \eta$ decay formfactors from light-cone sum rules. Phys. Rev. D 71, 014015 (2005). arXiv:hep-ph/0406232

75. A. V. Rusov, "Probing new physics in $b \rightarrow d$ transitions. arXiv:1911.12819 [hep-ph]
76. A.K. Alok, A. Dighe, S. Ray, CP asymmetry in the decays $B \rightarrow$ $\left(X_{s}, X_{d}\right) \mu^{+} \mu^{-}$with four generations. Phys. Rev. D 79, 034017 (2009). arXiv:0811.1186 [hep-ph]

77. H.M. Asatrian, K. Bieri, C. Greub, M. Walker, Virtual corrections and bremsstrahlung corrections to $b \rightarrow d l^{+} l^{-}$in the standard model. Phys. Rev. D 69, 074007 (2004). arXiv:hep-ph/0312063

78. S. Descotes-Genon, L. Hofer, J. Matias, J. Virto, Global analysis of $b \rightarrow s \ell \ell$ anomalies. JHEP 1606, 092 (2016). arXiv:1510.04239 [hep-ph]

79. B. Kindra and N. Mahajan, "Predictions of angular observables for $\bar{B}_{s} \rightarrow K^{*} \ell \ell$ and $\bar{B} \rightarrow \rho \ell \ell$ in the standard model", Phys. Rev. D 98, no. 9, 094012 (2018). arXiv:1803.05876 [hep-ph]

80. W. Altmannshofer, P. Ball, A. Bharucha, A.J. Buras, D.M. Straub, M. Wick, Symmetries and asymmetries of $B \rightarrow K^{*} \mu^{+} \mu^{-}$decays in the standard model and beyond. JHEP 0901, 019 (2009). arXiv:0811.1214 [hep-ph]

81. G. Hiller, M. Schmaltz, Diagnosing lepton-nonuniversality in $b \rightarrow$

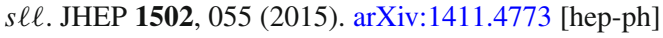

82. A.J. Buras, J. Girrbach-Noe, C. Niehoff, D.M. Straub, $B \rightarrow$ $K^{(*)} \nu \bar{v}$ decays in the standard model and beyond. JHEP 02, 184 (2015). arXiv:1409.4557 [hep-ph]

83. J. Grygier et al. [Belle], "Search for $\boldsymbol{B} \rightarrow \boldsymbol{h} \boldsymbol{v} \overline{\boldsymbol{v}}$ decays with semileptonic tagging at Belle", Phys. Rev. D 96, no.9, 091101 (2017). arXiv:1702.03224 [hep-ex]

84. J. Lees et al. [BaBar], "Search for $B \rightarrow K^{(*)} v \bar{v}$ and invisible quarkonium decays", Phys. Rev. D 87, no. 11, 112005 (2013). arXiv:1303.7465 [hep-ex]

85. O. Lutz et al. [Belle], "Search for $B \rightarrow h^{(*)} v \bar{v}$ with the full Belle $\Upsilon(4 S)$ data sample", Phys. Rev. D 87, no. 11, 111103 (2013). arXiv:1303.3719 [hep-ex]

86. P. del Amo Sanchez et al. [BaBar], "Search for the rare decay $B \rightarrow K v \bar{v}$ ", Phys. Rev. D 82, 112002 (2010). arXiv:1009.1529 [hep-ex]

87. J. Aebischer, J. Kumar, P. Stangl and D. M. Straub, "A global likelihood for precision constraints and flavour anomalies", Eur. Phys. J. C 79, no. 6, 509 (2019). arXiv: 1810.07698 [hep-ph]

88. A. A. Petrov, Charm mixing in the standard model and beyond, Int. J. Mod. Phys. A 21, 5686-5693 (2006). arXiv:hep-ph/0611361 [hep-ph]

89. E. Golowich, J. Hewett, S. Pakvasa, A.A. Petrov, Relating D0-antiD0 mixing and D0 $->1+1$ - with new physics. Phys. Rev. D 79, 114030 (2009). arXiv:0903.2830 [hep-ph]

90. N. Carrasco et al. [ETM], " $\mathrm{S}=2$ and $\mathrm{C}=2$ bag parameters in the standard model and beyond from $\mathrm{N}_{f}=2+1+1$ twisted-mass lattice QCD”, Phys. Rev. D 92 (2015) no. 3, 034516. arXiv:1505.06639 [hep-lat]

91. E. Golowich, J. Hewett, S. Pakvasa, A.A. Petrov, Implications of $D^{0}-\bar{D}^{0}$ Mixing for new physics. Phys. Rev. D 76, 095009 (2007). arXiv:0705.3650 [hep-ph]

92. G. Burdman, E. Golowich, J. L. Hewett, S. Pakvasa, "Rare charm decays in the standard model and beyond", Phys. Rev. D 66, 014009 (2002). arXiv:hep-ph/0112235 [hep-ph]

93. J. Lees et al., [BaBar], "Search for the decay $D^{0}->\gamma \gamma$ and measurement of the branching fraction for $D^{0}->\pi^{0} \pi^{0}$ ". Phys. Rev. D 85, 091107 (2012). arXiv:1110.6480 [hep-ex]

94. R. Aaij et al., [LHCb], "Search for the rare decay $D^{0} \rightarrow \mu^{+} \mu^{-"}$. Phys. Lett. B 725, 15-24 (2013). arXiv:1305.5059 [hep-ex] 